\title{
Dual meson condensates in the Polyakov-loop enhanced linear sigma model
}

\author{
Zhao Zhang $\odot,{ }^{*}$ Yunshuo Wang, and Haipeng Lu \\ School of Mathematics and Physics, North China Electric Power University, Beijing 102206, China
}

(Received 17 November 2017; revised 6 September 2020; accepted 26 January 2021; published 23 February 2021)

\begin{abstract}
The extent to which the dual meson condensates obtained in the Polyakov-loop enhanced linear sigma model can indicate deconfining transition is investigated by imposing the twisted boundary conditions. The influence of the improved unquenching effect, the dependence on the glue potential parametrization, and the role of the fermion vacuum contribution are focused on at the mean field level. At zero density, the rapid rise of the dual sigma condensate with $T$ is confirmed, which is more sensitive to the chiral transition than the increase of the Polyakov loop. For finite isospin chemical potential $\mu_{I}>m_{\pi} / 2$, the dual sigma condensate shows abnormal thermal behavior which decreases with $T$ below the melting temperature $T_{c}^{I_{3}}$ of pion superfluidity. In contrast, the dual pion condensate always increases with $T$, with the maximum slope located at $T_{c}^{I_{3}}$ rather than $T_{c}^{P}$, as determined by the Polyakov loop. The dual vector meson condensate for $\mu_{I}>m_{\pi} / 2$ is also more sensitive to the chiral restoration when considering the fermion vacuum contribution. The study suggests that the dual condensates calculated in this model are not appropriate indicators of deconfinement due to some limitations and uncertainty.
\end{abstract}

DOI: $10.1103 /$ PhysRevD.103.034017

\section{INTRODUCTION}

Understanding the confinement-deconfinement phase transition at finite temperature and density is a very important task in high-energy nuclear physics. However, it is conceptually difficult to define a relevant order parameter in QCD. So far, describing the deconfinement transition is still a subtle problem.

In the heavy quark limit, the expectation value of the Polyakov loop is the true order parameter for deconfinement, which is directly related to the center symmetry. Usually, the Polyakov loop is also extensively used to indicate the quark deconfining transition in lattice QCD (LQCD) [1-4] and effective models [5], even though the center symmetry is explicitly broken by dynamical quarks. Besides the Polyakov loop, some other quantities or criteria are also proposed and used to determine the deconfinement transition in the literature. These include the QCD monopole in the dual Ginzburg-Landau theory [6], the center vortex [7], the Polyakov-loop fluctuation [8], the entropy in the framework of a hybrid model [9], the quark number holonomy based on the topological picture $[10,11]$, the proposed order parameter accessible with functional methods [12], and so on.

\footnotetext{
*zhaozhang@pku.org.cn
}

Published by the American Physical Society under the terms of the Creative Commons Attribution 4.0 International license. Further distribution of this work must maintain attribution to the author(s) and the published article's title, journal citation, and DOI. Funded by SCOAP .
Inspired by the original paper [13], the dressed Ployakov loop (DPL) is suggested as an appropriate order parameter for deconfinement in QCD [14,15]. This quantity is defined as the first Fourier moment of the quark condensate obtained under the twisted boundary condition for quarks. In the lattice gauge theory language, the DPL includes contributions of infinite closed loops with winding number 1 around the temporal direction. So, it transforms in the same way as the Polyakov loop under the center transformation. (The Polyakov loop only includes the shortest loop contribution.) For infinite quark masses, the DPL reduces to a PL, since the spacial fluctuations are suppressed. ${ }^{1}$

One merit of the DPL is that it interpolates between the quark condensate and Polyakov loop, which may imply some intrinsic relation between chiral transition and deconfinement. Another is that it can also be calculated in some effective theories or models of QCD. The previous investigations in LQCD [14-16], the truncated DysonSchwinger equations (tDSE) [18-21], and Nambu-JonaLasinio (NJL)-type models [22-24] all indicate that the DPL exhibits order-parameter-like behavior, just as the Polyakov loop does. The coincidence of two phase transitions, namely $T_{c}^{\chi} \approx T_{c}^{D}$, is obtained in these studies.

Note that in QCD with light flavors, the explicit chiral symmetry breaking is small, since the current masses of $u$ and $d$ quarks are quite small compared to the QCD scale,

\footnotetext{
${ }^{1}$ One can construct many dual observables which belong to the same class as the Polyakov loop under the center transformation $[16,17]$.
} 
but the explicit center symmetry breaking is large. This means that in the real world, the quark condensate is a good order parameter, but whether the DPL is also a good one is unclear. There exists the possibility that the DPL is only sensitive to chiral restoration but insensitive to deconfining transition. So it is interesting to check to what extent the DPL can indicate the deconfinement.

Model studies may shed some light on this question. A particularly noticeable calculation [24] is that the DPL obtained in NJL is very similar to the lattice result: it increases with $T$ and changes rapidly near $T_{c}^{\chi}$. Since NJL is a well-known chiral model without gluon fields, such a rapid rise should have little relation with center symmetry. Actually, a Ginzburg-Landau analysis [25] manifests that it is totally driven by chiral transition. The NJL variants with different confining elements are also used to study the DPL $[26,27]$. It is found that the rapid rise of the DPL has no effect on the change of confining properties of the quark propagator [26]. By considering gluon degrees of freedom with center symmetry, it is confirmed that the rapid rise of the DPL is still determined by chiral restoration rather than the increase of the Polyakov loop [27], where PNJL [28,29] is used. ${ }^{2}$ It is also shown in Ref. [27] that for $\mu_{I}>m_{\pi} / 2$, the dual pion condensate (DPC) behaves similarly to the Polyakov loop, while the DPL decreases with $T$ until the pion condensate melts away. All of these seem to suggest that the DPL calculated in NJL-type models should not be regarded as the deconfining order parameter.

This raises a question: Is the DPL merely sensitive to chiral transition in QCD? If so, using this quantity to conclude the coincidence of the chiral and deconfinement transitions should be problematic. In this sense, it is necessary to first check whether the above NJL conclusion also holds in other QCD models, especially those with hadron degrees of freedom. On the other hand, the quark backreaction on the Polyakov-loop potential is only roughly included in the PNJL calculation [27]. We note that an improved Polyakov-loop potential with unquenching effect had been proposed based on the functional renormalization method of QCD [30-36]. It is interesting to study whether the main results in Ref. [27] are still robust when including the quark backreaction effect. Given that, the main purpose of this work is to try to extract the DPL and other dual quantities in the Polyakov-loop augmented linear sigma model (PL $\sigma \mathrm{M})$ of QCD (also known as PQM) by taking into account the quark-improved glue potential and comparing it with the previous NJL results [25-27].

$\mathrm{PL} \sigma \mathrm{M}$ [37] is a popular chiral model which has been extensively used to explore the QCD phase transitions. Different from PNJL, this model includes three types of degrees of freedom: quarks, mesons, and gluons.

\footnotetext{
${ }^{2}$ The dual quark condensate in PNJL is first calculated by Kashiwa in Ref. [22], where the role of vector interaction is addressed.
}

The philosophy of PL $\sigma \mathrm{M}$ is that quarks and gluons are relevant objects for $T>T_{c}$, while mesons play the dominant role in low temperatures. Compared to NJL, the $\mathrm{L} \sigma \mathrm{M}$ part in PL $\sigma \mathrm{M}$ has the merit of renormalizability. It is argued that $\mathrm{PL} \sigma \mathrm{M}$ is more suitable to study the QCD phase diagram than PNJL at low baryon density [5]. In the literature, $(\mathrm{P}) \mathrm{L} \sigma \mathrm{M}$ is also frequently employed to study the inhomogeneous chiral condensates at high baryon density [38] and the chiral transition in a magnetic field [39]. However, this model is seldom used to study the physics at imaginary chemical potential.

Since $\mathrm{L} \sigma \mathrm{M}$ can be viewed as a partially bosonized version of NJL in a certain sense, the dual observables related to some quark bilinears may be assessed indirectly through studying the corresponding meson condensates in $(\mathrm{P}) \mathrm{L} \sigma \mathrm{M}$ by imposing the twisted boundary conditions. In this article, the DPL and DPC mentioned above are evaluated in PL $\sigma \mathrm{M}$ by researching the dual sigma and pion condensates at the mean field level. Beyond Ref. [27], the dual vector meson condensate related to the isospin density is also calculated.

Unlike (P)NJL, the Dirac-sea contribution is not necessary for the dynamical chiral symmetry breaking in (P) $\mathrm{L} \sigma \mathrm{M}$. There exists subtlety on how to treat this term in (P) $\mathrm{L} \sigma \mathrm{M}$, which is ignored in Refs. [37,40] but taken into account in Ref. [41]. In our calculation, both treatments are adopted and compared.

The paper is organized as follows: In Sec. II, the dual meson condensates for both zero $\mu_{I}$ and $\mu_{I}>m_{\pi} / 2$ in $\mathrm{PL} \sigma \mathrm{M}$ are introduced, where the twisted boundary condition is used. The numerical results and discussion are given in Sec. III. In Sec. IV, we summarize.

\section{DUAL CONDENSATES IN PL $\sigma$ M WITH TWISTED BOUNDARY CONDITION}

\section{A. Two-flavor PL $\sigma$ M at finite $\mu$ and $\mu_{I}$}

We adopt the following Lagrangian density of the twoflavor PL $\sigma \mathrm{M}$ [42]:

$$
\begin{aligned}
\mathcal{L}= & \bar{q} S_{0}^{-1} q+\frac{1}{2}\left(\partial_{\mu} \sigma\right)^{2}+\frac{1}{2}\left(\partial_{\mu} \vec{\pi}\right)^{2}-U(\sigma, \vec{\pi}) \\
& -\frac{1}{4} \omega^{\mu \nu} \omega_{\mu \nu}-\frac{1}{4} \vec{R}^{\mu \nu} \vec{R}_{\mu \nu}+\frac{1}{2} m_{v}^{2}\left(\omega^{\mu} \omega_{\mu}+\vec{R}^{\mu} \cdot \vec{R}_{\mu}\right) \\
& -\mathcal{U}(\Phi, \bar{\Phi}, T),
\end{aligned}
$$

with

$$
S_{0}^{-1}=i \gamma^{\mu} D_{\mu}-g\left(\sigma+i \gamma_{5} \vec{\tau} \cdot \vec{\pi}\right)-g_{\omega} \gamma^{\mu} \omega_{\mu}-g_{\rho} \gamma^{\mu} \vec{\tau} \cdot \vec{R}_{\mu},
$$

where $q$ denotes the quark field, $\vec{\tau}$ is the Pauli matrix in the flavor space,

$$
D^{\mu}=\partial^{\mu}-i A^{\mu}, \quad \text { and } \quad A^{\mu}=\delta_{0}^{\mu} A^{0},
$$


with $A^{0}=-i A_{4}$. The gauge coupling $\mathrm{g}$ is absorbed in $A^{\mu}(x)=\mathrm{g} A_{a}^{\mu}(x) \frac{\lambda_{a}}{2}$, where $A_{a}^{\mu}(x)$ is the gluon field and $\lambda_{a}$ are the Gell-Mann matrices. The mesonic potential $U$ is given as

$$
U(\sigma, \vec{\pi})=\lambda\left(\sigma^{2}+\vec{\pi}^{2}-v^{2}\right)^{2} / 4-h \sigma,
$$

wherein $\sigma$ and $\vec{\pi}$ are the isoscalar-scalar and isovectorpseudoscalar meson fields. The vector meson degrees of freedom are also taken into account, and $\omega^{\mu \nu}$ and $\vec{R}^{\mu \nu}$ are the field tensors of $\omega$ and $\rho$ mesons, respectively. The term $\mathcal{U}(\Phi, \bar{\Phi}, T)$ is the Polyakov-loop potential, which respects the $Z(3)$ center symmetry. Here $\Phi\left[\left\langle A_{4}\right\rangle\right]$ is the Polyakov-loop variable, and $\bar{\Phi}$ is the conjugate of $\Phi$.

In PL $\sigma \mathrm{M}$, the quark chemical potential $\mu$ is introduced in the same way as in QCD. However, the introduction of $\mu_{I}$ is quite different. Under the isospin $U(1)_{I_{3}}$ transformation, the quark and pion fields change in the following way:

$q \rightarrow e^{i \tau_{3} \theta} q, \quad q^{\dagger} \rightarrow e^{-i \tau_{3} \theta} q^{\dagger}, \quad \pi_{ \pm} \rightarrow e^{\mp i 2 \theta} \pi_{ \pm}$.

The corresponding conserved current takes the form

$$
J_{\mu}^{3}=\bar{q} \tau_{3} \gamma_{\mu} q+2 i\left(\pi_{-} \partial_{\mu} \pi_{+}-\pi_{+} \partial_{\mu} \pi_{-}\right),
$$

where

$$
\pi_{ \pm}=\pi_{1} \pm i \pi_{2}
$$

Accordingly, $\mu_{I}$ can be introduced by adding the term $\mu_{I} I^{3}$ to the Hamiltonian, where the associated conserved charge is

$$
I^{3}=\int d^{3} \vec{x}\left(\bar{q} \tau_{3} \gamma_{0} q+\pi_{1} \partial_{t} \pi_{2}-\pi_{2} \partial_{t} \pi_{1}\right) .
$$

The Lagrangian density [Eq. (1)] is then modified at finite $\mu$ and $\mu_{I}$ by the following replacements:

$$
S_{0}^{-1} \rightarrow S_{0}^{-1}+\gamma_{0} \hat{\mu}
$$

and

$\left(\partial_{\mu} \vec{\pi}\right)^{2} \rightarrow\left(\partial_{\mu} \pi_{0}\right)^{2}+\left(\left(\partial_{\mu}+2 \mu_{I} \delta_{\mu}^{0}\right) \pi_{+}\right)\left(\partial_{\mu}+2 \mu_{I} \delta_{\mu}^{0}\right) \pi_{-}$,

where

$$
\hat{\mu}=\left(\begin{array}{ll}
\mu_{u} & \\
& \mu_{d}
\end{array}\right)=\left(\begin{array}{ll}
\mu+\mu_{I} & \\
& \mu-\mu_{I}
\end{array}\right) .
$$

The reason for the appearance of $\mu_{I}^{2} \pi_{+} \pi_{-}$in Eq. (10) is that the generalized momenta of pion fields have been integrated out according to the standard derivation [43].

The phase diagram of a two-flavor $\mathrm{L} \sigma \mathrm{M}$ at finite $\mu, \mu_{I}$, and $T$ has been investigated in Ref. [44], where the pion superfluid phase is also studied. Note that the effects of the Polyakov-loop dynamics and vector mesons are all ignored in that work. Taking into account these elements and following the treatment in Ref. [45], we derive the mean field thermal potential of PL $\sigma \mathrm{M}$ at finite $\mu$ and $\mu_{I}$ :

$$
\begin{aligned}
\Omega= & -2 N_{c} \int \frac{d^{3} p}{(2 \pi)^{3}}\left[E_{p}^{-}+E_{p}^{+}\right] \theta\left(\Lambda^{2}-\vec{p}^{2}\right)-2 T \int \frac{d^{3} p}{(2 \pi)^{3}}\left\{\ln \left[1+3\left(\Phi+\bar{\Phi} \mathrm{e}^{-\left(E_{p}^{-}-\mu^{\prime}\right) \beta}\right) \mathrm{e}^{-\left(E_{p}^{-}-\mu^{\prime}\right) \beta}+\mathrm{e}^{-3\left(E_{p}^{-}-\mu^{\prime}\right) \beta}\right]\right. \\
& +\ln \left[1+3\left(\bar{\Phi}+\Phi \mathrm{e}^{-\left(E_{p}^{-}+\mu^{\prime}\right) \beta}\right) \mathrm{e}^{-\left(E_{p}^{-}+\mu^{\prime}\right) \beta}+\mathrm{e}^{-3\left(E_{p}^{-}+\mu^{\prime}\right) \beta}\right]+\ln \left[1+3\left(\Phi+\bar{\Phi} \mathrm{e}^{-\left(E_{p}^{+}-\mu^{\prime}\right) \beta}\right) \mathrm{e}^{-\left(E_{p}^{+}-\mu^{\prime}\right) \beta}+\mathrm{e}^{-3\left(E_{p}^{+}-\mu^{\prime}\right) \beta}\right] \\
& \left.+\ln \left[1+3\left(\bar{\Phi}+\Phi \mathrm{e}^{-\left(E_{p}^{+}+\mu^{\prime}\right) \beta}\right) \mathrm{e}^{-\left(E_{p}^{+}+\mu^{\prime}\right) \beta}+\mathrm{e}^{-3\left(E_{p}^{+}+\mu^{\prime}\right) \beta}\right]\right\}-2 \mu_{I} \pi^{2}-\frac{1}{2}\left(M_{\omega}^{2} \omega^{2}+M_{\rho}^{2} R^{2}\right)+U(\sigma, \pi)+\mathcal{U}(\Phi, \bar{\Phi}, T),
\end{aligned}
$$

with the quasiparticle energy $E_{p}^{ \pm}=\sqrt{\left(E_{p} \pm \mu_{I}^{\prime}\right)^{2}+N^{2}}$ and $E_{p}=\sqrt{\vec{p}^{2}+M^{2}}$, in which the two energy gaps are defined as

$$
\begin{aligned}
& M=g \sigma, \\
& N=g \pi .
\end{aligned}
$$

Here (also in the following), $\sigma$ and $\pi$ refer to the vacuum expectation values (VEVs) of the sigma and charged pion mesons, and the latter is defined as

$$
\pi=\left\langle\pi_{+}\right\rangle e^{i \theta^{\prime}}=\left\langle\pi_{-}\right\rangle e^{-i \theta^{\prime}} .
$$

Nonzero $\pi$ indicates the spontaneous breaking of the $U(1)_{I_{3}}$ symmetry, and the phase factor $\theta^{\prime}$ is the breaking direction. $\mu^{\prime}$ and $\mu_{I}^{\prime}$ are the shifted quark and isospin chemical potentials:

$$
\mu^{\prime}=\mu-g_{\omega} \omega, \quad \mu_{I}^{\prime}=\mu_{I}-g_{\rho} \rho,
$$

where $\omega$ and $\rho$ denote the VEVs of $\omega$ and $\rho_{0}$ mesons,

$$
\omega=\left\langle\omega_{0}\right\rangle, \quad \rho=\left\langle R_{0}^{3}\right\rangle,
$$

respectively. Following Ref. [42], the momentum cutoff $\Lambda$ is used in Eq. (12). 
In this paper, we only consider the situation with finite $\mu_{I}$ and vanishing $\mu$. In this case, $\Phi$ is strictly equal to $\bar{\Phi}[45]$, and it is free from the sign problem even in the lattice simulation. The reason for the latter is that $\tau_{2} \gamma_{5} D \gamma_{5} \tau_{2}=D^{\dagger}$, which ensures $\operatorname{det} D \geq 0$ [46], where $D$ is the Dirac operator. Minimizing the thermal dynamical potential [Eq. (12)], the motion equations for the mean fields $\sigma, \pi, \Phi$, and $\rho$ are determined by

$\frac{\partial \Omega}{\partial \sigma}=0, \quad \frac{\partial \Omega}{\partial \pi}=0, \quad \frac{\partial \Omega}{\partial \Phi}=0, \quad \frac{\partial \Omega}{\partial \rho}=0$.

This set of equations is then solved for the fields $\sigma, \pi, \Phi$, and $\rho$ as functions of $T$ and $\mu_{I}$.

\section{B. Two-flavor PL $\sigma \mathrm{M}$ at finite $\mu_{I}$ with twisted boundary condition}

To calculate the dual observables, we must adopt the twisted boundary condition in the time direction for quarks:

$$
q(x, \beta=1 / T)=e^{i \phi} q(x, 0),
$$

where $\phi$ ranges from zero to $2 \pi$. Under this condition, the modified quark chemical potential $\mu^{\prime}$ in Eq. (12) should be replaced by $i T(\phi-\pi)$ [14,17,22], which is nothing but an imaginary baryon chemical potential. There is no sign problem for purely imaginary baryon chemical potential, since

$$
\operatorname{det} D(\mu)=\operatorname{det}\left[\gamma_{5} D(\mu) \gamma_{5}\right]=\operatorname{det}^{*} D\left(-\mu^{*}\right) .
$$

For details on lattice simulations at finite $\mu_{I}$ and imaginary $\mu$, please refer to Refs. [47,48].

Strictly speaking, $\mu^{\prime}$ at $\phi \neq \pi$ should contain an imaginary part $g_{\omega} \omega$, even when $\mu$ is zero. ${ }^{3}$ Such a term is ignored in our calculation. It has been shown in Ref. [22] that a similar term in PNJL has little effect on DPL near $T_{c}^{\chi}$. Note that $\mu_{I}^{\prime}$ is always real because the imaginary parts of $\mu_{u}^{\prime}$ and $\mu_{d}^{\prime}$ cancel each other out. This means that $\rho$ is still real for $\phi \neq \pi$. This quantity resembles the isospin density in NJL with vector interactions [27].

In the standard definition of DPL $[14,15]$, the twisted boundary condition is imposed on the Dirac operator $D_{\phi}$, and the bracket $\langle\cdots\rangle$ still keeps the antiperiodic condition with $\phi=\pi$. So in our calculation, $\Phi$ as a function of $T$ and $\mu_{I}$ is first obtained by solving Eq. (18) using the physical boundary condition. ${ }^{4}$ The other quantities, such as $\sigma(\phi)$,

\footnotetext{
${ }^{3}$ In PL $\sigma \mathrm{M}, \omega$ is closely related to the dual density proposed in Ref. [17].

${ }^{4}$ Note that to calculate the DPL, the Roberge-Weiss periodicity-namely $\Omega(\theta)=\Omega(\theta+2 \pi / 3)$ at finite imaginary chemical potential $\mu=i \theta T$-must be broken explicitly; otherwise, the DPL always remains zero $[17,49]$. This might lead to some uncertainty on the DPL calculation (see related discussions in Ref. [49]).
}

$\pi(\phi)$, and $\rho(\phi)$ are then determined by the following coupled equations:

$$
\frac{\partial \Omega}{\partial \sigma(\phi)}=0, \quad \frac{\partial \Omega}{\partial \pi(\phi)}=0, \quad \frac{\partial \Omega}{\partial \rho(\phi)}=0,
$$

with $\Phi$ keeping its value for $\phi=\pi$. Such a treatment is consistent with Refs. [22,27].

\section{Dual meson condensates at finite $\mu_{I}$}

According to Ref. [14], the DPL is defined as

$$
\Sigma_{\sigma}^{(1)}=-\int_{0}^{2 \pi} \frac{d \phi}{2 \pi} e^{-i \phi}\langle\bar{q} q\rangle_{\phi},
$$

where $\langle\bar{q} q\rangle_{\phi}$ is the generalized quark condensate. Similarly, the dual pion condensate

$$
\Sigma_{\pi}^{(1)}=-\int_{0}^{2 \pi} \frac{d \phi}{2 \pi} e^{-i \phi}\left\langle\bar{q} i \gamma_{5} \tau_{1} q\right\rangle_{\phi}
$$

is introduced in Ref. [27]. Both of these (and also the dual density proposed in Ref. [17]) are gauge invariant, which means they merely include contributions of closed loops with winding number 1 . As mentioned, they belong to the same class as the Polyakov loop under the $Z(3)$ center transformation.

Following Eqs. (22) and (23), we can construct the dual sigma condensate $(\mathrm{D} \sigma \mathrm{C})$ and the dual pion condensate $(\mathrm{D} \pi \mathrm{C})$ in $\mathrm{PL} \sigma \mathrm{M}$, namely

$$
\Sigma_{\sigma}^{1}=\int_{0}^{2 \pi} \frac{d \phi}{2 \pi} e^{-i \phi} \sigma(\phi)
$$

and

$$
\Sigma_{\pi}^{1}=\int_{0}^{2 \pi} \frac{d \phi}{2 \pi} e^{-i \phi} \pi(\phi) .
$$

Since the VEVs of meson fields are gauge invariant, the first moments of $\sigma(\phi)$ and $\pi(\phi)$ also belong to the same class as PL under the center transformation. Evidently, the $\mathrm{D} \sigma \mathrm{C}$ and $\mathrm{D} \pi \mathrm{C}$ correspond to $\Sigma_{\sigma}^{(1)}$ and $\Sigma_{\pi}^{(1)}$, respectively. The main task of this work is to test whether these dual meson condensates could be used as order parameters for deconfinement in PL $\sigma \mathrm{M}$.

Besides the $\mathrm{D} \sigma \mathrm{C}$ and $\mathrm{D} \pi \mathrm{C}$, we can also define the dual vector meson condensate $(\mathrm{D} \rho \mathrm{C})$ in $\mathrm{PL} \sigma \mathrm{M}$, namely

$$
\Sigma_{\rho}^{1}=-\int_{0}^{2 \pi} \frac{d \phi}{2 \pi} e^{-i \phi} \rho(\phi) .
$$

This quantity is nonzero at finite $T$ and $\mu_{I}$ (or zero $T$ for $\left.\mu_{I}>m_{\pi} / 2\right)$. As mentioned, $\rho(\phi)$ corresponds to the isospin density $\left\langle\bar{q} \gamma_{0} \tau_{3} q\right\rangle_{\phi}$ in QCD or NJL with vector interactions. 
In this sense, $\Sigma_{\rho}^{1}$ is analogous to the dual density proposed in Ref. [17]. It is interesting to check whether this dual isospin density can be used to indicate the deconfining transition.

\section{Polyakov-loop potential with the quark backreaction effect}

We will focus on the improved unquenching effect on the dual meson condensates in PL $\sigma \mathrm{M}$ by comparing two types of Polyakov-loop potential which take into account the quark backreaction in different ways.

The first is that proposed early in Ref. [29] (we denote it as $\left.\mathcal{U}_{\text {old }}\right)$, which has been extensively used. The $\mathcal{U}_{\text {old }}$ is obtained by replacing the original parameter $T_{0}=$ $270 \mathrm{MeV}$ in the Yang-Mills Polyakov-loop potential $\mathcal{U}_{\text {YM }}$ with $T_{0} \sim 200 \mathrm{MeV}$. Such a rescaling, which was adopted just for comparison with the available LQCD data, was argued to be justified [37]: the presence of quarks leads to an $N_{f}$-dependent decrease of $\Lambda_{\mathrm{QCD}}$ at zero $T$, which translates into an $N_{f}$-dependent decrease of $T_{0}$ at finite $T$. In this sense, the rescaled $T_{0}\left(N_{f}\right)$ may have the uncertainty of tens of MeV [37]. Since the $\mathcal{U}_{\text {old }}$ only works well for the old LQCD data, we think that the quark backreaction effect is only roughly included in it.

Another type is the improved Polyakov-loop potential (we denote it as $\mathcal{U}_{\text {glue }}$ ) suggested in Ref. [32], which is based on the functional renormalization group method by incorporating the unquenching effect. It was found that the inclusion of quarks alters the whole glue potential but keeps its form as the function of the traced Polyakov loop [32]. This means that the $\mathcal{U}_{\text {glue }}$ can be mapped to the $\mathcal{U}_{\text {YM }}$ via a rescaling of the whole temperature scale in terms of the reduced temperature

$$
t=\frac{T-T_{c}}{T_{c}} .
$$

Namely, the improved glue potential $\mathcal{U}_{\text {glue }}$ is given by

$$
\frac{\mathcal{U}_{\text {glue }}}{T^{4}}\left(t_{\text {glue }}, \phi, \bar{\phi}\right)=\frac{\mathcal{U}_{\mathrm{YM}}}{T^{4}}\left(t_{\mathrm{YM}}, \phi, \bar{\phi}\right)
$$

through the relation

$$
t_{\mathrm{YM}}\left(t_{\text {glue }}\right)=0.57 t_{\text {glue }} \text {. }
$$

The $T_{c}^{\mathrm{YM}}$ in $\mathcal{U}_{\mathrm{YM}}$ is just the critical Yang-Mills temperature $T_{0}=270 \mathrm{MeV}$, and the $T_{c}^{\text {glue }}$ in $\mathcal{U}_{\text {glue }}$ needs to be determined by fitting the lattice data. In the PL $\sigma \mathrm{M}$ with $\mathcal{U}_{\text {glue }}$, the $T_{c}^{\text {glue }}$ is treated as an open parameter [30-33,35,36] in the range of $180 \mathrm{MeV} \leq T_{c}^{\text {glue }} \leq 270 \mathrm{MeV}$, which mainly depends on the choice of $m_{\sigma}$.

The $\mathcal{U}_{\text {old }}$ and $\mathcal{U}_{\text {glue }}$ mentioned above are all based on the parametrization of $\mathcal{U}_{\mathrm{YM}}$. The logarithmic and polynomial forms of $\mathcal{U}_{\mathrm{YM}}$ are quite popular in the literature (for more details, see Refs. [30,31]). It was shown in Ref. [30] that the logarithmic $\mathcal{U}_{\mathrm{YM}}$ is in the best agreement with the lattice data for the pure gauge theory, and the $2+1$ flavor PL $\sigma \mathrm{M}$ using the logarithmic $\mathcal{U}_{\text {glue }}\left(\mathcal{U}_{\text {glue }}^{\text {log }}\right)$ with $T_{c}^{\text {glue }}=210 \mathrm{MeV}$ and $m_{\sigma}=500 \mathrm{MeV}$ can reproduce the new LQCD data quite well (in the mean field level without the vacuum contribution). Note that the logarithmic $\mathcal{U}_{\text {old }}\left(\mathcal{U}_{\text {old }}^{\text {log }}\right)$ with $T_{0}=200 \mathrm{MeV}$ was adopted to calculate the DPL in the two-flavor PNJL [22,27]. For comparison, we mainly adopt the logarithmic potential with the same $T_{0} / T_{c}^{\text {glue }}$ in this paper.

To check the parametrization dependence, the $\mathrm{D} \sigma \mathrm{C}$ is also calculated using the polynomial $\mathcal{U}_{\text {glue }}\left(\mathcal{U}_{\text {glue }}^{\text {pol }}\right)$, which had been employed in the two-flavor PL $\sigma \mathrm{M}$ to calculate the QCD pressure and interaction measure in Ref. [31].

\section{E. Model parameters in the matter sector}

The parameters $g, \lambda, v^{2}$, and $h$ in the matter sector are specified to reproduce the following vacuum properties: $\sigma=f_{\pi}, m_{\pi}^{2}=h / f_{\pi}, m_{q}^{0}=g f_{\pi}$, and $m_{\sigma}^{2}=\frac{\partial^{2} \Omega_{\mathrm{MF}}}{\partial \sigma^{2}}$, where $m_{\pi}=138 \mathrm{MeV}, f_{\pi}=93 \mathrm{MeV}$, and $m_{q}^{0}=300 \mathrm{MeV}$ are adopted according to Refs. [30,31,40]. We mainly focus on $m_{\sigma}=600 \mathrm{MeV}$ in this paper. For simplicity, $g_{\omega}=g_{\rho}$ is assumed, ${ }^{5}$ which is fixed as $0.25 \mathrm{~g}$. We have checked that our main conclusion is insensitive to $g_{\rho}$. As in Ref. [42], we also assume the common vector meson masses $\left(m_{\omega}=m_{\rho}=770 \mathrm{MeV}\right)$.

We will perform calculations in the standard (extended) mean field level without (with) the fermion vacuum loop contribution. In the standard mean field (sMF) calculation, $\lambda=\left(m_{\sigma}^{2}-m_{\pi}^{2}\right) /\left(2 f_{\pi}^{2}\right)$ and $v^{2}=f_{\pi}^{2}-h /\left(\lambda f_{\pi}\right)$ [40]. In the extended mean field (eMF) study, parameters $\lambda$ and $v^{2}$ depend on the regularization method. Two methodsnamely, the direct UV momentum cutoff and the dimensional regularization-are employed and compared for zero $\mu_{I}$ in the eMF calculations. We choose the "standard" momentum cutoff $\Lambda=600 \mathrm{MeV}$ in the first method. ${ }^{6}$ The corresponding parameters $\lambda$ and $v^{2}$ for $m_{\sigma}=600 \mathrm{MeV}$ are fixed as 2.02 and $-(0.649)^{2} \mathrm{GeV}^{2}$, respectively. In the second method, the renormalized vacuum contribution

$$
\Omega_{q \bar{q}}^{\mathrm{reg}}=-\frac{N_{c} N_{f}}{8 \pi^{2}} m_{q}^{4} \ln \left(\frac{m_{q}}{M}\right)
$$

is included in the thermodynamical potential [41], where $M$ is an arbitrary renormalization scale parameter.

\footnotetext{
${ }^{5}$ In general, $g_{\omega}$ is different from $g_{\rho}$, which may lead to flavor $\underset{6}{\operatorname{mixing}}$ at finite $\mu_{I}[50]$.

${ }^{6}$ It was found that the results using dimensional regularization can be reproduced quite well by using the sharp momentum cutoff $\Lambda=600 \mathrm{MeV}$ [44,51].
} 


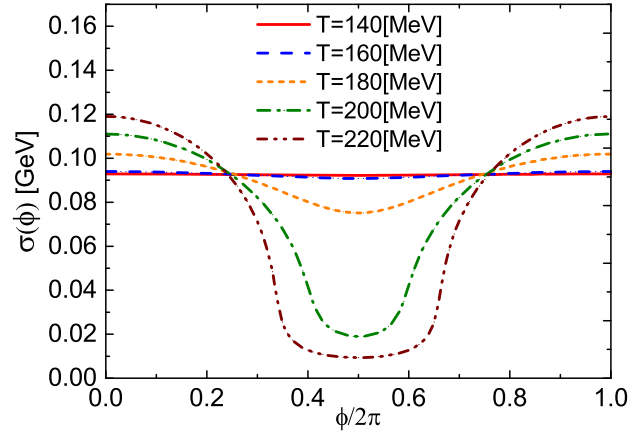

(a)

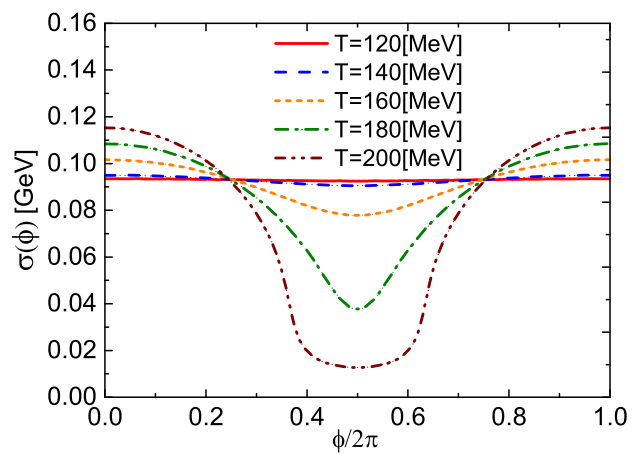

(b)

FIG. 1. The sMF results for the twisted angle dependence of the sigma condensate $\sigma$ at zero $\mu_{I}$ and different temperatures in the two-flavor PL $\sigma \mathrm{M}$ with $m_{\sigma}=600 \mathrm{MeV}$. Panels (a) and (b) show results for cases a and b, respectively: cases a and b correspond to sMF calculations using $\mathcal{U}_{\text {old }}^{\log }$ with $T_{0}=200 \mathrm{MeV}$ and $\mathcal{U}_{\text {glue }}^{\log }$ with $T_{c}^{\text {glue }}=210 \mathrm{MeV}$, respectively.

The parameters $\lambda$ and $v^{2}$ are all $M$-dependent in this method, namely

$\lambda=\frac{9 f_{\pi}^{3} g^{4}-2 h \pi^{2}+2 f_{\pi} m_{\sigma}^{2} \pi^{2}+12 f_{\pi}^{3} g^{4} \ln \left(\frac{f_{\pi} g}{M}\right)}{4 f_{\pi}^{3} \pi^{2}}$,

$v^{2}=\frac{2 f_{\pi}^{2}\left(3 f_{\pi}^{3} g^{4}-3 h \pi^{2}+f_{\pi} m_{\sigma}^{2} \pi^{2}\right)}{9 f_{\pi}^{3} g^{4}-2 h \pi^{2}+2 f_{\pi} m_{\sigma}^{2} \pi^{2}+12 f_{\pi}^{3} g^{4} \ln \left(\frac{f_{\pi} g}{M}\right)}$,

but the mean-field physical thermodynamical potential is $M$-independent.

To check the $m_{\sigma}$-dependence, following Ref. [30], we also adopt $m_{\sigma}=400,500 \mathrm{MeV}$ in the sMF calculations by using $\mathcal{U}_{\text {glue }}^{\log }$. Note that in the eMF study, we find that the sigma condensate has no real solution for $\phi$ near $2 \pi$ or 0 once $m_{\sigma}<500 \mathrm{MeV}$. This is why we do not show the eMF results for a small $m_{\sigma}$ in this paper.

\section{NUMERICAL RESULTS AND DISCUSSIONS}

In Secs. III A and III B, the dual meson condensates at zero and finite $\mu_{I}$, respectively, are studied in detail, where $m_{\sigma}=600 \mathrm{MeV}$ is chosen and the logarithmic glue potentials $\mathcal{U}_{\text {old }}^{\text {log }}$ with $T_{0}=200 \mathrm{MeV}$ and $\mathcal{U}_{\text {glue }}^{\text {log }}$ with $T_{c}^{\text {glue }}=210 \mathrm{MeV}$ are adopted. The dependence on the parametrization of the glue potential is given in Sec. III C. In Sec. III D, we investigate the dependence on the sigma meson mass. The discussion is given in Sec. III E.

\section{A. Dual sigma condensate for zero $\mu_{I}$ in PL $\sigma \mathrm{M}$}

The thermal properties of the $\mathrm{D} \sigma \mathrm{C}$ and its relation with the sigma condensate and the expectation value of the traced Polyakov loop are first investigated at zero $\mu_{I}$ in the two-flavor PL $\sigma \mathrm{M}$. The following six cases are focused on: cases a and $\mathrm{b}$ correspond to the sMF calculations using $\mathcal{U}_{\text {old }}^{\log }$ and $\mathcal{U}_{\text {glue }}^{\text {log }}$, respectively, while cases c and d (cases e and f) correspond to the eMF calculations with the UV cutoff (dimensional regularization) method using $\mathcal{U}_{\text {old }}^{\text {log }}$ and $\mathcal{U}_{\text {glue }}^{\text {log }}$, respectively.

\section{1. $\phi$-dependence of the sigma condensate}

Figure 1 shows the sMF results for the sigma condensate as a function of the twisted angle $\phi$ at different temperatures. Panels (a) and (b) in Fig. 1 correspond to cases a and b, respectively. Figure 2 shows the eMF results for the sigma condensate as a function of $\phi$ at different temperatures. Panels (c)-(f) in Fig. 2 correspond to cases c-f, respectively.

As expected, both Figs. 1 and 2 display that the sigma condensate does not exhibit the Roberge-Weiss periodicity. All panels in Figs. 1 and 2 show that at low temperatures, $\sigma$ is insensitive to $\phi$ and the line of $\sigma(\phi)$ almost keeps flat; with increasing $T, \sigma$ decreases in the fermion-like region $(\phi \sim \pi)$ but increases in the boson-like region $(\phi \sim 0$ or $2 \pi)$. These features are qualitatively consistent with those of the generalized quark condensate obtained in LQCD [14], the truncated Dyson-Schwinger method [19], and PNJL $[22,27]$. On the other hand, Fig. 2 shows that the sigma condensate obtained using the UV cutoff $\Lambda=600 \mathrm{MeV}$ is almost quantitatively consistent with that obtained using dimension regularization when the same glue potential is adopted.

\section{Thermal property of the dual sigma condensate}

The $\mathrm{sMF}(\mathrm{eMF})$ results for the dual sigma condensate $\Sigma_{\sigma}^{1}$, the Polyakov-loop expectation value $\Phi$, and the sigma condensate $\sigma$ and $T$-derivatives of these quantities as functions of $T$ are shown in Figs. 3 and 4 (Figs. 5 and 6), respectively, which are obtained under the same conditions as adopted in Fig. 1 (Fig. 2). In the following, $A^{\prime}$ is used to denote the $T$-derivative of the quantity $A$.

Both Figs. 3 and 5 indicate that $\Sigma_{\sigma}^{1}$ increases monotonically with $T$, which remains quite small at low temperature and raises rapidly in the chiral transition region, no matter 


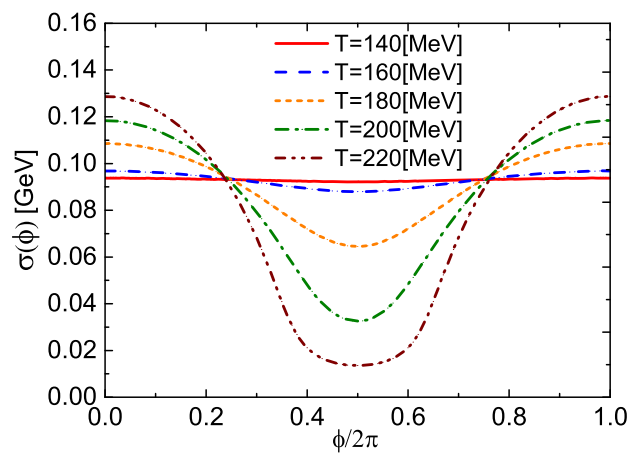

(c)

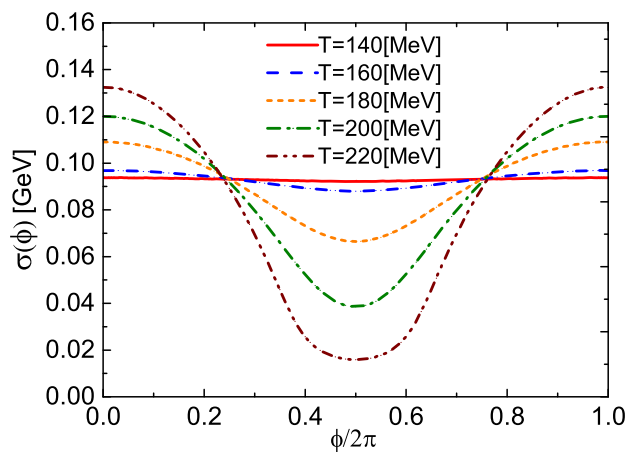

(e)

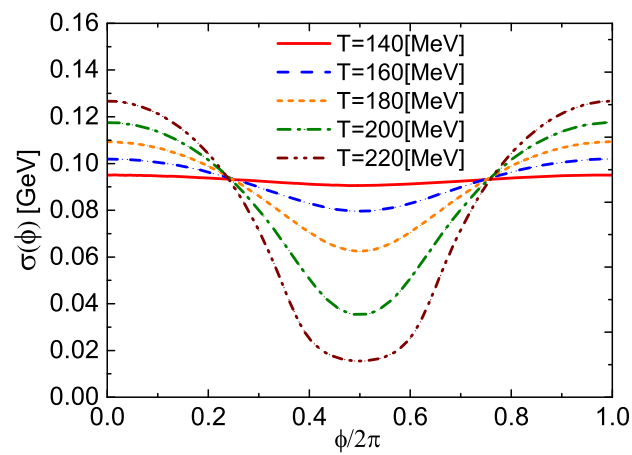

(d)

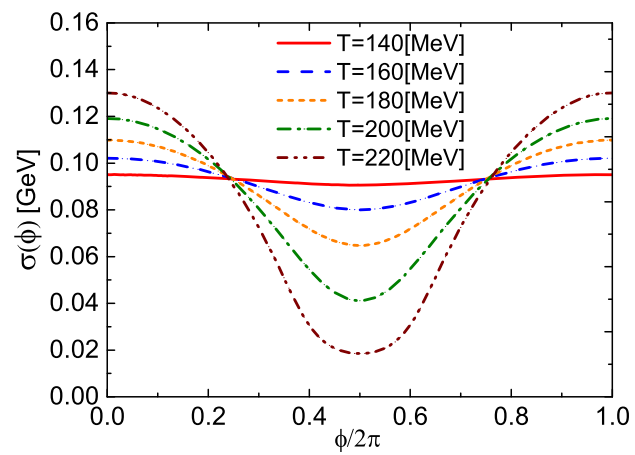

(f)

FIG. 2. The eMF results for the twisted angle dependence of the sigma condensate $\sigma$ at zero $\mu_{I}$ and different temperatures in the twoflavor PL $\sigma \mathrm{M}$ with $m_{\sigma}=600 \mathrm{MeV}$. Panels (c)-(f) show results for cases c-f, respectively: cases c and d (e and f) correspond to eMF calculations using the UV cutoff $\Lambda=600 \mathrm{MeV}$ (dimensional regularization) by adopting $\mathcal{U}_{\text {old }}^{\log }$ with $T_{0}=200 \mathrm{MeV}$ and $\mathcal{U}_{\text {glue }}^{\log }$ with $T_{c}^{\text {glue }}=210 \mathrm{MeV}$, respectively.

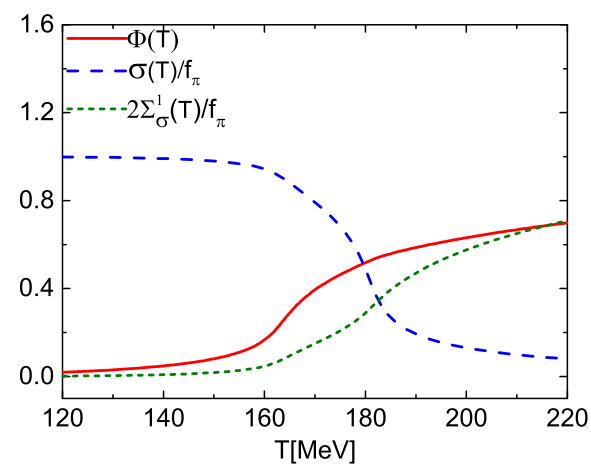

(a)

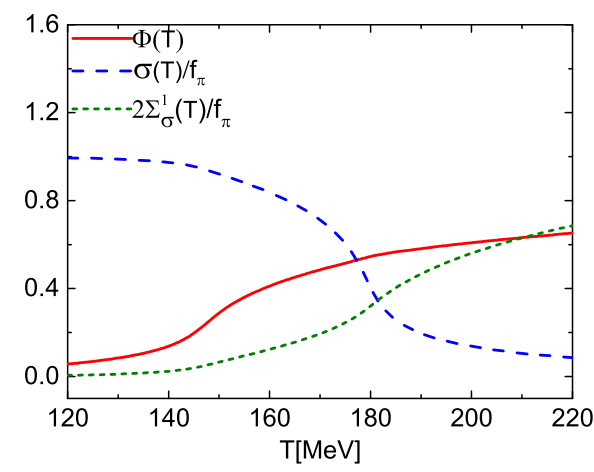

(b)

FIG. 3. The sMF results for the Polyakov loop, the sigma condensate, and the dual sigma condensate as functions of $T$ for zero $\mu_{I}$ in the two-flavor PL $\sigma \mathrm{M}$. Panels (a) and (b) correspond to cases a and b described in the caption of Fig. 1, respectively.

whether the Dirac sea contribution and/or the improved unquenching effect are included or not. This implies that at zero $\mu_{I}$, the $\mathrm{D} \sigma \mathrm{C}$ calculated in PL $\sigma \mathrm{M}$ really behaves like the DPL obtained in LQCD and other methods.

We use the $T$-derivative maximum of an order parameter to identify the crossover critical temperature. Both panels in Fig. 4 show that $\Phi^{\prime},-\sigma^{\prime}$, and $\Sigma_{\sigma}^{1 \prime}$ each have double peaks: one is at or near the deconfining crossover temperature $T_{c}^{P}$, and another is at the chiral crossover temperature $T_{c}^{\chi}$. Here, $T_{c}^{P}$ and $T_{c}^{\chi}$ are determined by the maximum slopes of $\Phi$ and $-\sigma$, respectively. In contrast to Figs. 4(a) and 4(b) indicates that both the chiral and deconfining transitions become milder and the difference between $T_{c}^{\chi}$ and $T_{c}^{P}$ gets larger owing to the improved unquenching effect. 


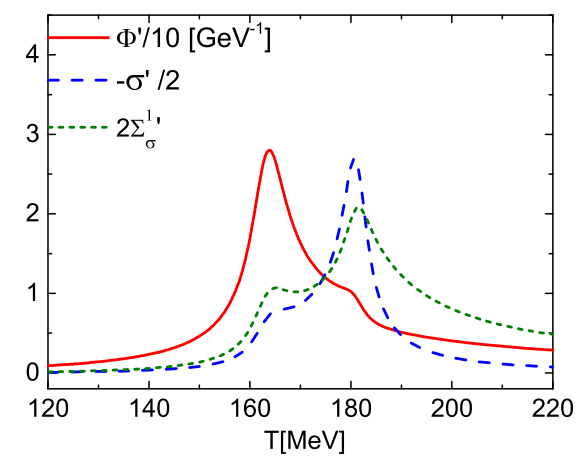

(a)

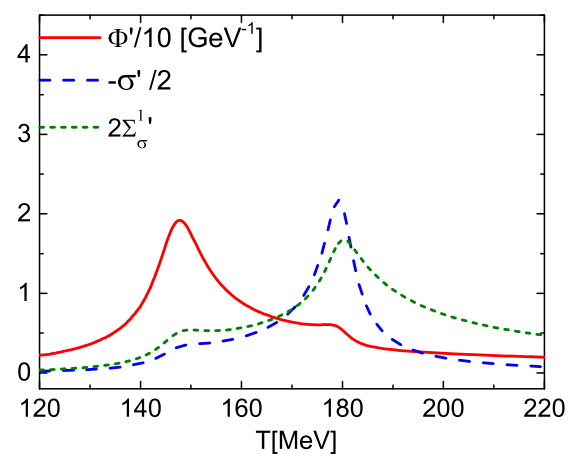

(b)

FIG. 4. Similar to Fig. 3, but for T-derivatives of the Polyakov loop, the sigma condensate, and the dual sigma condensate as functions of $T$.

We use $T_{c}^{d \sigma}$ to denote the position of the maximum of $\Sigma_{\sigma}^{1 \prime}$. Both panels in Fig. 4 clearly show that $T_{c}^{d \sigma} \approx T_{c}^{\chi}$. This means that the $\mathrm{D} \sigma \mathrm{C}$ changes more swiftly near $T_{c}^{\chi}$ rather than $T_{c}^{P}$, as demonstrated in Fig. 3. Actually, we see that the $T$-dependence of $\Sigma_{\sigma}^{1 \prime}$ is more similar to that of $-\sigma^{\prime}$ than to that of $\Phi^{\prime}$. This suggests that the $\mathrm{D} \sigma \mathrm{C}$ is more sensitive to chiral transition, and thus it seems that using it as a deconfining order parameter is questionable. One may argue that the first small peak in $\Sigma_{\sigma}^{1 \prime}$ can be used to indicate

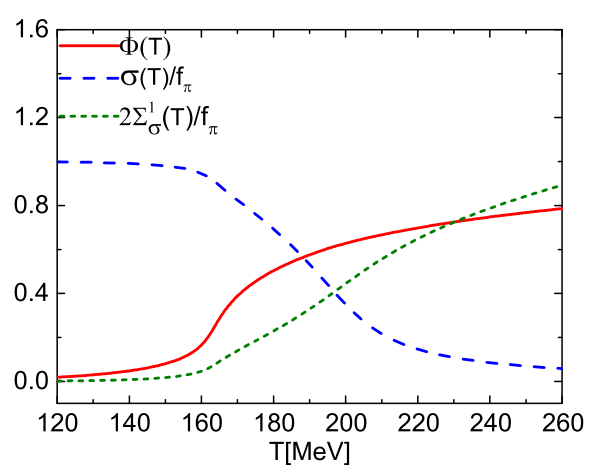

(c)

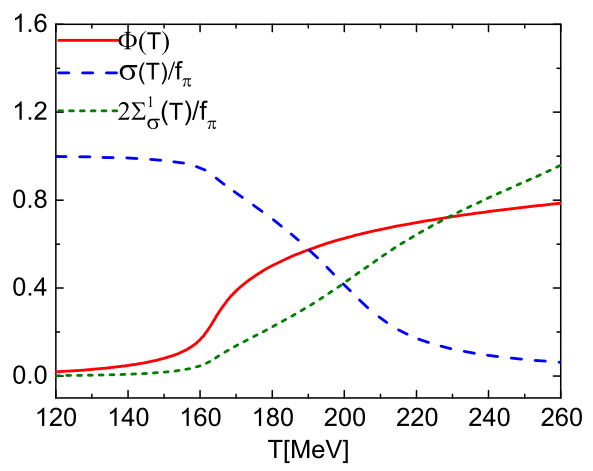

(e) the deconfining pseudocritical temperature $T_{c}^{P}$. Since the small peak in $-\sigma^{\prime}$ can do this too, it seems that there is no reason to regard the $\Sigma_{\sigma}^{1}$ as the deconfining order parameter. In other words, the $\mathrm{D} \sigma \mathrm{C}$ provides no more information on deconfining transition compared to the sigma condensate.

We then turn to eMF calculations. Figures 5 and 6 show that under the same condition, the results using the UV cutoff accord quite well with that using dimensional regularization, especially in the low-temperature region:

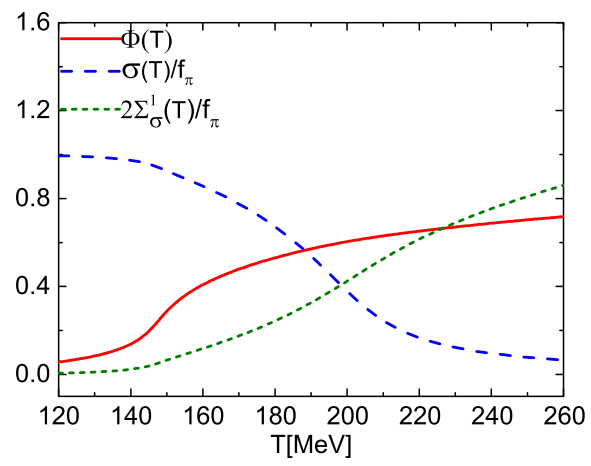

(d)

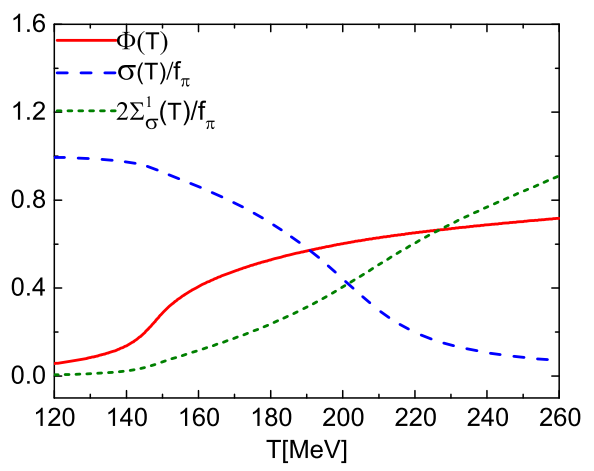

(f)

FIG. 5. The eMF results for the Polyakov loop, the sigma condensate, and the dual sigma condensate as functions of $T$ for zero $\mu_{I}$ in the two-flavor PL $\sigma$ M. Panels (c)-(f) correspond to cases c-f described in the caption of Fig. 2, respectively. 


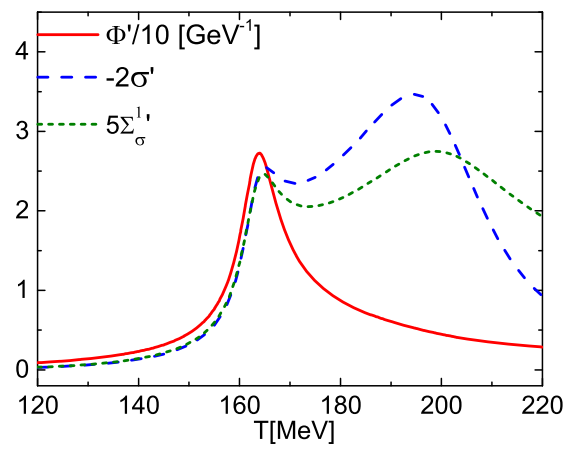

(c)

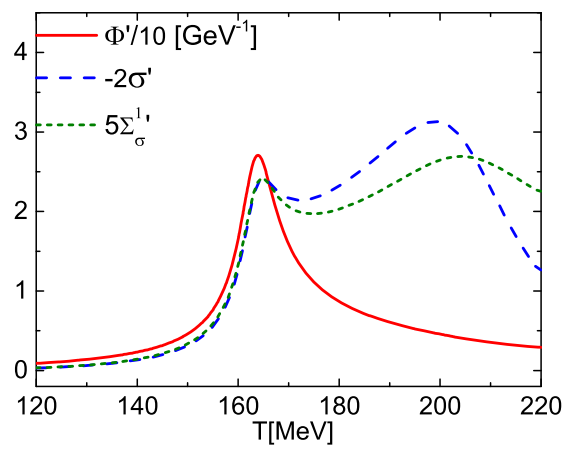

(e)

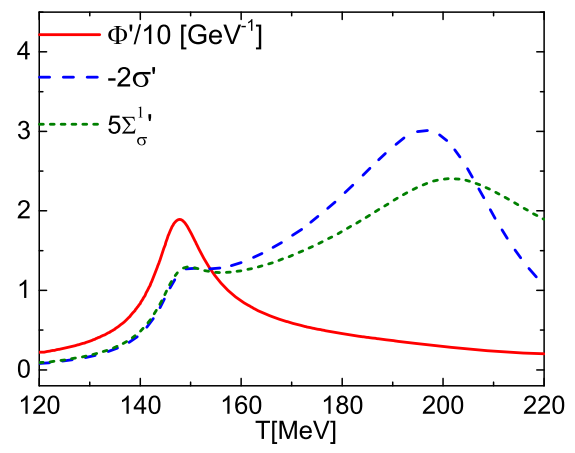

(d)

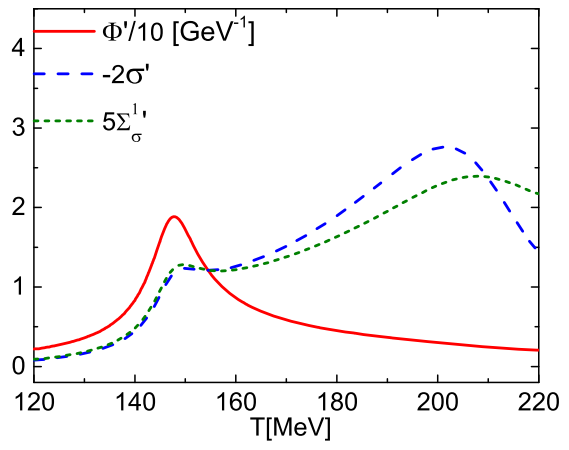

(f)

FIG. 6. Similar to Fig. 5, but for $T$-derivatives of the Polyakov loop, the sigma condensate, and the dual sigma condensate as functions of $T$.

Both methods give almost the same $T_{c}^{P}$, and the error for the crossover $T_{c}^{\chi}$ due to the cutoff is within $3 \%$ compared to the cutoff-independent result. This supports the claim in Refs. [44,51] that the eMF calculation with a sharp $600 \mathrm{MeV}$ cutoff was found to well reproduce results using dimensional regularization. Figure 5 shows that the $\mathrm{D} \sigma \mathrm{C}$ also exhibits a similar $T$-dependence to the Polyakov loop. Figure 6 displays that $-\sigma \prime$ and $\Sigma_{\sigma}^{1 \prime}$ each still have double peaks, but $\Phi^{\prime}$ has only one. This change owes to the smoother chiral transition due to the fermion vacuum contribution. Compared to Figs. 4 and 6 displays that the $T_{c}^{\chi}$ is rather higher, which is $\sim 200 \mathrm{MeV}$, and the separation of chiral and deconfining transitions is quite large $\left(T_{c}^{\chi}-T_{c}^{P} \sim 35-50 \mathrm{MeV}\right)$. Note that Fig. 6 indicates that the $T$-dependence of $\Sigma_{\sigma}^{1 \prime}$ is still very similar to that of $-\sigma^{\prime}$, and thus $T_{c}^{d \sigma} \approx T_{c}^{\chi}$. Both Figs. 5 and 6 show that the deconfining crossover becomes broader owing to the improved unquenching effect. Compared to the case using $\mathcal{U}_{\text {old }}^{\text {log }}$, we see that the peak of $\Sigma_{\sigma}^{1 \prime}$ near $T_{c}^{P}$ is obviously lower than that near $T_{c}^{\chi}$ when $\mathcal{U}_{\text {glue }}^{\log }$ is used under the same condition. This is consistent with the sMF results shown in Fig. 4.

In PL $\sigma \mathrm{M}$, the difference between $T_{c}^{\chi}$ and $T_{c}^{P}$ can become smaller by choosing a small $m_{\sigma}$, which can lead to a steeper chiral transition [30]. Thus, $T_{c}^{d \sigma} \approx T_{c}^{\chi}$ should still hold for a smaller $m_{\sigma}$, since decreasing $m_{\sigma}$ has a mild influence on the Polyakov loop. The $m_{\sigma}$-dependence will be addressed in Sec. III D. Of course, when $m_{\sigma}$ is small enough, the chiral and deconfining transitions may coincide. In this paper, we do not consider such a special case with (almost) degenerate chiral and Ployakov-loop critical temperatures. Actually, the new lattice study suggests that the Polyakov loop still remains quite small $(\sim 0.1)$ near $T_{c}^{\chi}$, and the pseudocritical temperature $T_{c}^{P}$ determined by the lattice Polyakov loop is significantly larger than $T_{c}^{\chi}$.

The coincidence of $T_{c}^{d \sigma}$ and $T_{c}^{\chi}$ in both sMF and eMF calculations implies that the rapid increase of $\mathrm{D} \sigma \mathrm{C}$ with $T$ is mainly driven by the swift drop of $\sigma$ rather than the increase of $\Phi$. In this sense, it might be not appropriate to regard the $\mathrm{D} \sigma \mathrm{C}$ as the deconfining order parameter, at least in PL $\sigma \mathrm{M}$. Such a conclusion is robust when considering the improved quark backreaction effect in this model, since the deconfining transition is softened.

Moreover, we confirm that the $\mathrm{D} \sigma \mathrm{C}$ obtained in $\mathrm{L} \sigma \mathrm{M}$ shows a similar $T$-dependence to that in PL $\sigma \mathrm{M}$, even though the center symmetric glue potential is not considered. In this case, the only peak of the $T$-derivative of the $\mathrm{D} \sigma \mathrm{C}$ is located exactly at $T_{c}^{\chi}$ in the chiral limit, which is similar to the DPL slope calculated in NJL [25]. This implies that the rapid rise of $\mathrm{D} \sigma \mathrm{C}$ in $\mathrm{L} \sigma \mathrm{M}$ is also totally driven by chiral transition. Since the $\mathrm{D} \sigma \mathrm{C}$ in $(\mathrm{P}) \mathrm{L} \sigma \mathrm{M}$ is 


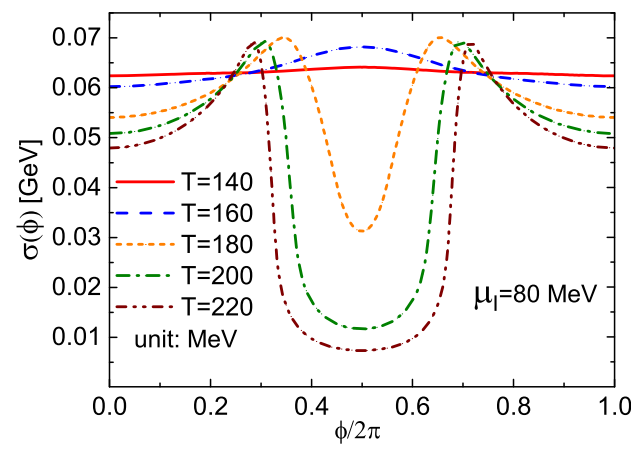

(a)

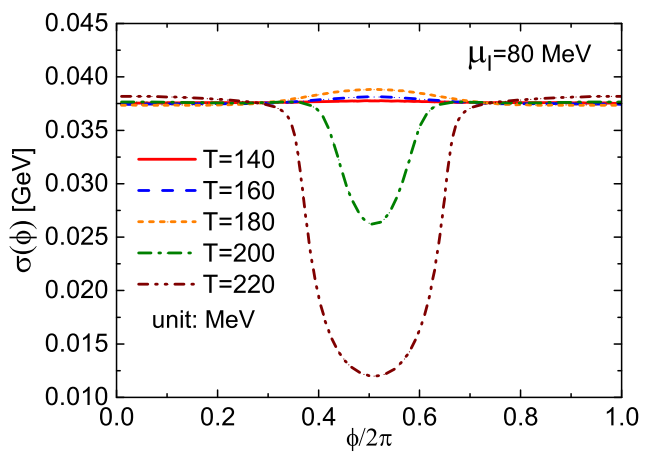

(c)

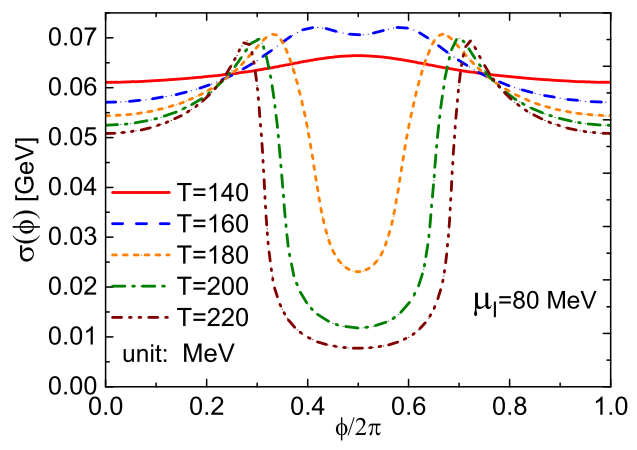

(b)

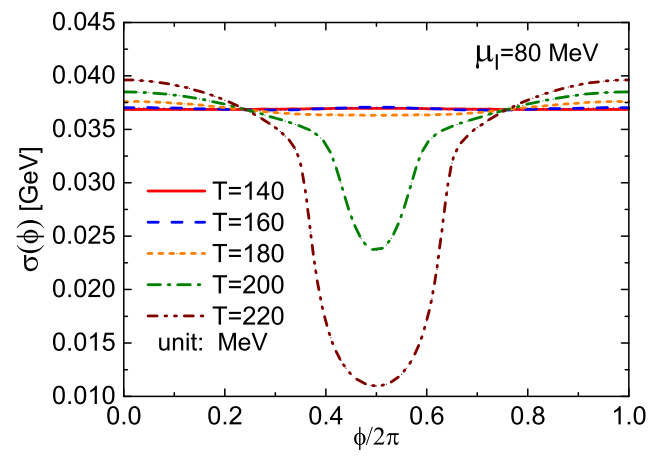

(d)

FIG. 7. Twisted angle dependences of the sigma condensate $\sigma$ at $\mu_{I}=80 \mathrm{MeV}$ for different temperatures in the two-flavor PL $\sigma \mathrm{M}$ with $m_{\sigma}=600 \mathrm{MeV}$. Panels (a)-(d) correspond to cases a-d, respectively. The meanings of cases a-d are same as those described in the captions of Figs. 1 and 2.

the counterpart of the DPL in (P)NJL, we conclude that the main result in Refs. $[25,26]$ that the DPL obtained in NJL-type models without center symmetry only reflects the chiral transition is also supported in $\mathrm{L} \sigma \mathrm{M}$ with the quark field. Moreover, Figs. 3-6 demonstrate that such a conclusion is still valid in PL $\sigma \mathrm{M}$.

\section{B. Dual meson condensates for nonzero $\mu_{I}$ in PL $\sigma \mathrm{M}$}

We then extend the study to $\mu_{I}>m_{\pi} / 2$ to check how the $\mathrm{D} \sigma \mathrm{C}$ evolves with $T$ when the sigma condensate is suppressed by the pion condensate in PL $\sigma \mathrm{M}$. In addition, thermal properties of the $\mathrm{D} \pi \mathrm{C}$ and $\mathrm{D} \rho \mathrm{C}$ are also investigated. The cases a-d mentioned in Sec. III A are focused on here. Since the dimensional regularization cannot be used directly in the presence of the pion condensate, we choose only the UV cutoff method in the eMF calculations. The cutoff $\Lambda=600 \mathrm{MeV}$ is still chosen, since it works quite well at zero $\mu_{I}$. In the following, $T_{c}^{I_{3}}$ is used to denote the melting temperature of pion superfluidity.

\section{1. $\phi$-dependences of the meson condensates}

The generalized meson condensates $\sigma, \pi$, and $\rho$ as functions of $\phi$ in cases a-d at $\mu_{I}=80 \mathrm{MeV}$ for different temperatures are shown in Figs. 7-9, respectively. ${ }^{7}$

Figure 7 displays that in the fermion-like region, $\sigma(\phi)$ is a concave line for $T>T_{c}^{I_{3}}$ but a convex one for $T<T_{c}^{I_{3}}$, no matter whether the vacuum term is included or not. This is distinct with Figs. 1 and 2, where only concave curves emerge. The difference can be traced back to the fact that $\sigma(\phi \sim \pi)$ first increases and then decreases ${ }^{8}$ with $T$ near $T_{c}^{I_{3}}$. Moreover, Fig. 7 shows that the thermal behavior of $\sigma$ in the boson-like region is sensitive to the fermion vacuum

\footnotetext{
${ }^{7}$ The reason for choosing $\mu_{I}=80 \mathrm{MeV}$ in our calculations is that in a quite narrow window around this value, the sigma condensate is still comparable to the pion condensate $T<T_{c}^{I_{3}}$ even if the former is suppressed obviously by the latter. For $\mu_{I} \gg m_{\pi} / 2$, we confirm that the sigma condensate becomes negligible for $T<T_{c}^{I_{3}}$ and the melting of pion condensate becomes very steep, which is similar to the PNJL result [45]; the maximum slope of the dual pion condensate is still located near $T_{c}^{I_{3}}$.

${ }^{8}$ This is also observed in Ref. [45] and other chiral model studies [52,53]. The reason for such an anomaly is that compared to the decline of $\sqrt{\sigma^{2}+\pi^{2}}, \pi$ drops more significantly with $T$ near $T_{c}^{I_{3}}$.
} 


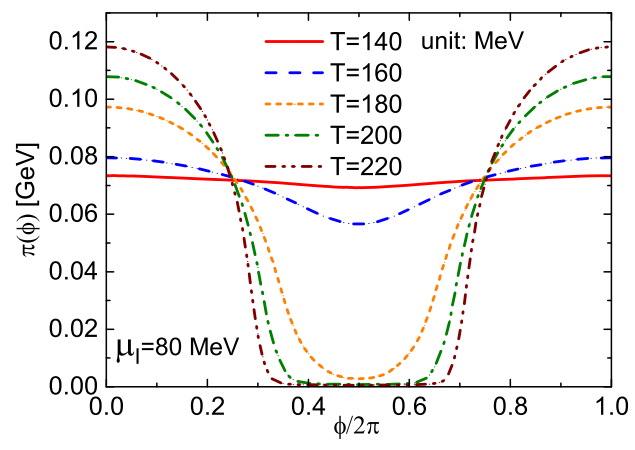

(a)

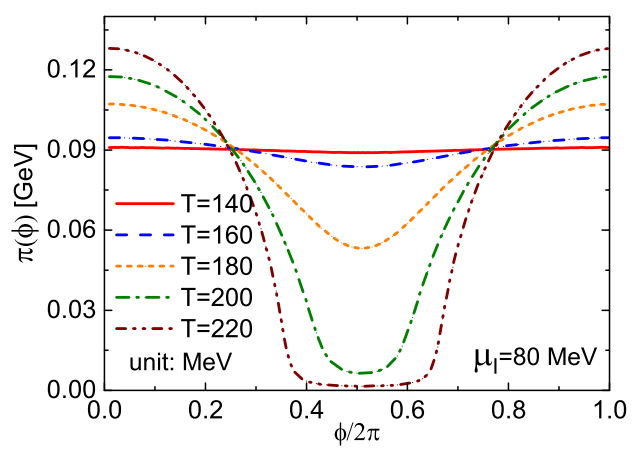

(c)

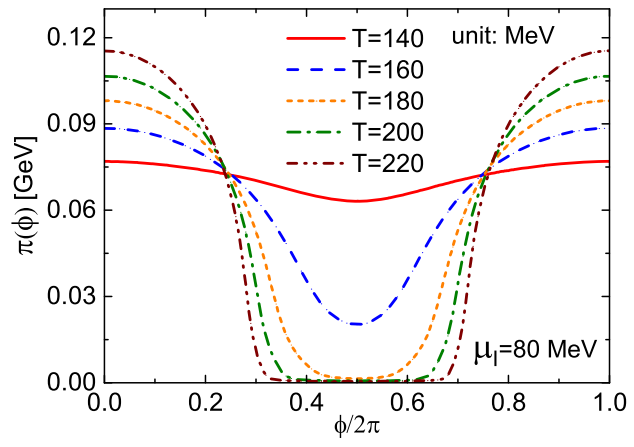

(b)

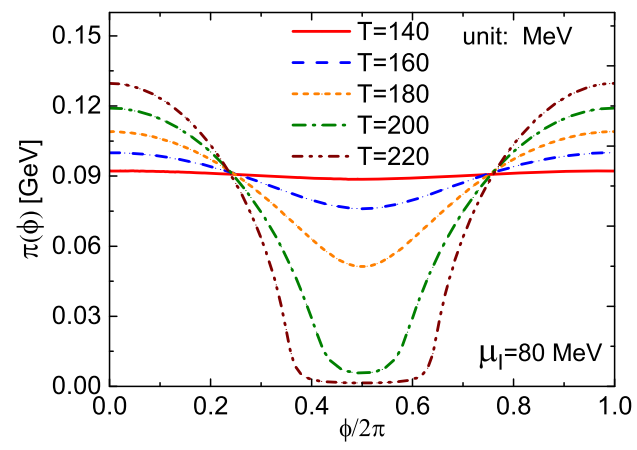

(d)

FIG. 8. Similar to Fig. 7, but for the twisted angle dependences of the pion condensate $\pi$.

term: it decreases (increases) with $T$ without (with) the fermion vacuum term.

Figure 8 shows that the $\phi$-dependence of $\pi$ is analogous to that of $\sigma$ displayed in Figs. 1 and 2. The similarity can be understood in this way: For $\mu_{I}>m_{\pi} / 2$, the sigma condensate partially turns into the pion condensate, and thus the latter inherits some properties of the former. Consequently, such a transformation leads to an obvious modification of $\sigma(\phi)$, as demonstrated in Fig. 7.

The $\phi$-dependence of the density $\rho$ is shown in Fig. 9. We see that this quantity is also insensitive to $\phi$ at low temperatures. All panels in Fig. 9 display that with increasing $T, \rho$ decreases near $\phi \sim \pi$ but increases around $\phi \sim 0$. Thus, only convex lines appear in Fig. 9.

Note that as functions of $\phi$, the condensates $\pi$ and $\sigma$ in PL $\sigma \mathrm{M}$ with the vacuum contribution are qualitatively consistent with the corresponding PNJL results in Ref. [27].

\section{Thermal behaviors of dual meson condensates}

The Polyakov loop, the sigma and pion condensates, the dual sigma and pion condensates, and the dual isospin density as functions of temperature at $\mu_{I}=80 \mathrm{MeV}$ are shown in Fig. 10, where panels (a)-(d) correspond to cases $\mathrm{a}-\mathrm{d}$, respectively. The $T$-derivatives of these quantities are displayed in Fig. 11.

Figure 10 shows that the $T$-dependences of the Polyakov loop and of sigma and pion condensates are all analogous to those obtained in PNJL [45]. In particular, with increasing $T$, the sigma condensate almost keeps unchanged or even increases slowly up to $T_{c}^{I_{3}}$ and then declines. As mentioned, the unnatural thermal behavior of $\sigma$ is due to the fast dropping of $\pi$ [52,53]. Figure 10 indicates that the $\mathrm{D} \pi \mathrm{C}$ and $\mathrm{D} \rho \mathrm{C}$ always increase with $T$, which resemble the Polyakov loop. In contrast, panels (a)-(c) display that the $\mathrm{D} \sigma \mathrm{C}$ first decreases with $T$ up to $T_{c}^{I_{3}}$ and then increases, which is quite different from the Polyakov loop; Panel (d) shows that the D $\sigma \mathrm{C}$ keeps almost unchanged for $T<T_{c}^{I_{3}}$ and increases quickly with $T$ for $T>T_{c}^{I_{3}}$. Note that in case $\mathrm{d}$, the $\mathrm{D} \sigma \mathrm{C}$ first decreases (slowly) with $T$, as is also observed for a smaller $\mu_{I}$.

The abnormal $T$-dependence of $\Sigma_{\sigma}^{1}$ can be directly attributed to the nonconcave lines of $\sigma(\phi)$ displayed in Fig. 7, or the unusual $T$-dependence of $\sigma$ mentioned above. Figure 10 clearly shows that when $\sigma$ increases with $T, \Sigma_{\sigma}^{1}$ decreases, and vice versa. This gives further evidence that $\Sigma_{\sigma}^{1}$ is quite sensitive to $\sigma$ but not $\Phi$ (at least in this model), since the latter always increases with $T$. Actually, panels (c) and (d) show that the absolute value of $\Sigma_{\sigma}^{1}$ remains very small in the temperature range $T_{c}^{P}<T<T_{c}^{I_{3}}$ where $\Phi$ has already become large enough. Such an anomaly is also in agreement with the PNJL result [27], where the DPL exhibits similar thermal behavior.

Accordingly, the maximum slope of $\Sigma_{\sigma}^{1}$ is still located around $T_{c}^{\chi}$, as shown in Fig. 11. Figure 11(a) displays that 


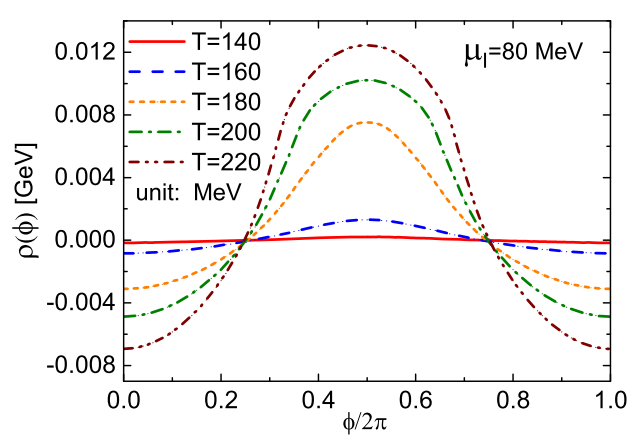

(a)

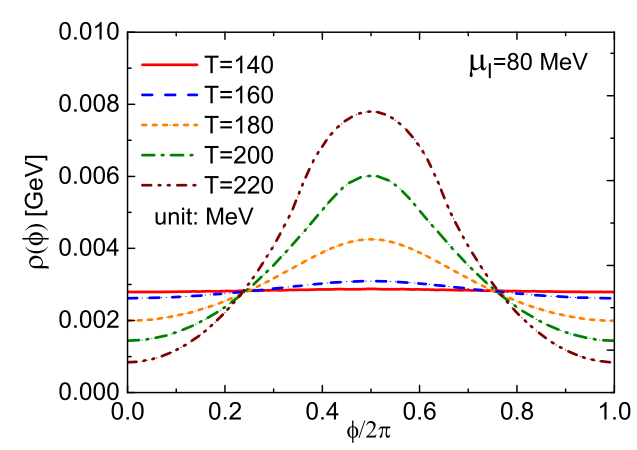

(c)

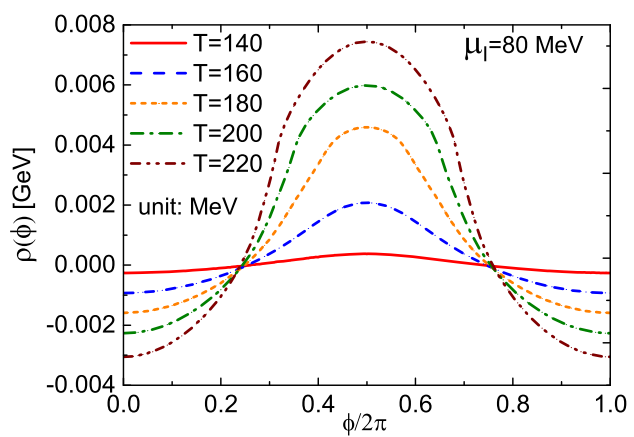

(b)

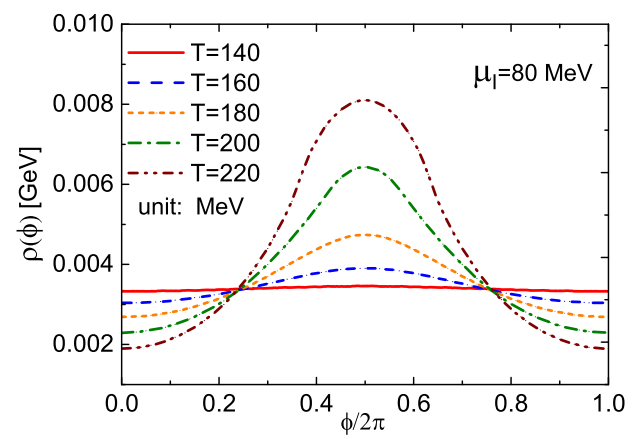

(d)

FIG. 9. Similar to Fig. 7, but for the twisted angle dependences of the isospin density $\rho$.

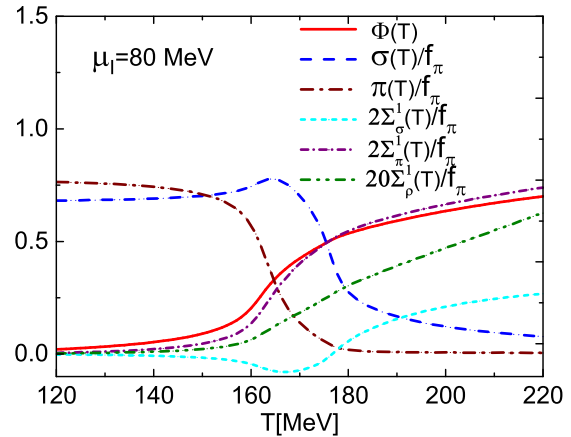

(a)

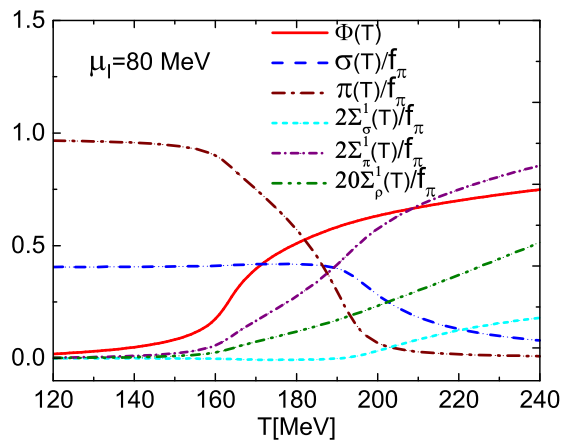

(c)

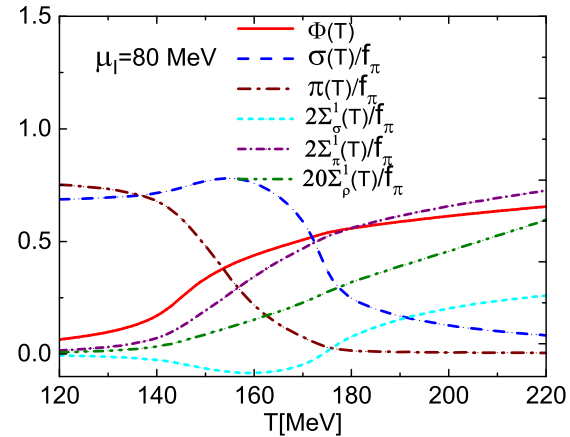

(b)

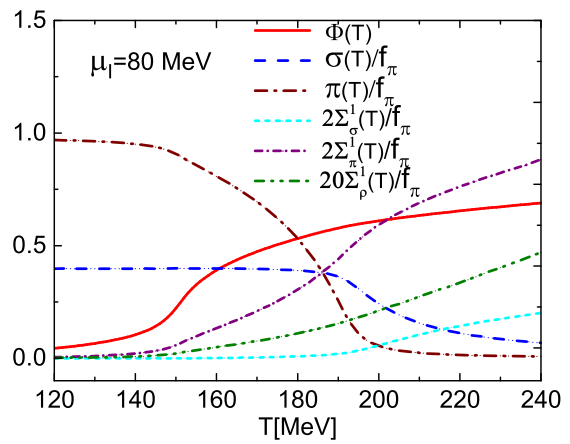

(d)

FIG. 10. The Polyakov loop, the sigma and pion condensates, the dual sigma and pion condensates, and the dual isospin density as functions of $T$ at $\mu_{I}=80 \mathrm{MeV}$ in the two-flavor PL $\sigma \mathrm{M}$ with $m_{\sigma}=600 \mathrm{MeV}$. Panels (a)-(d) correspond to cases a-d, respectively. The meanings of cases a-d are same as those described in the captions of Figs. 1 and 2. 


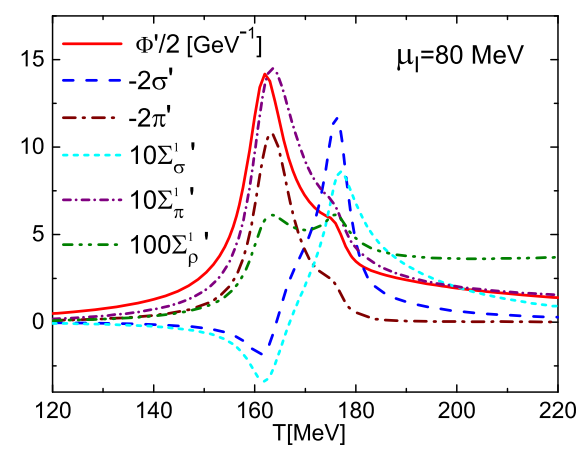

(a)

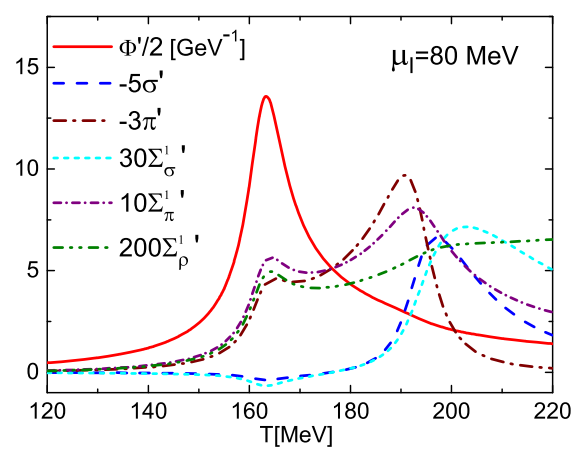

(c)

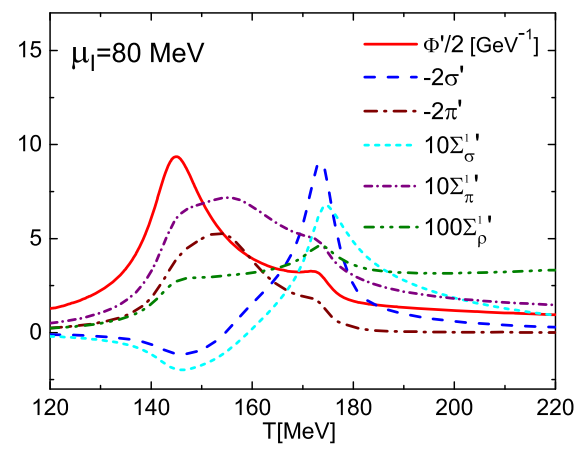

(b)

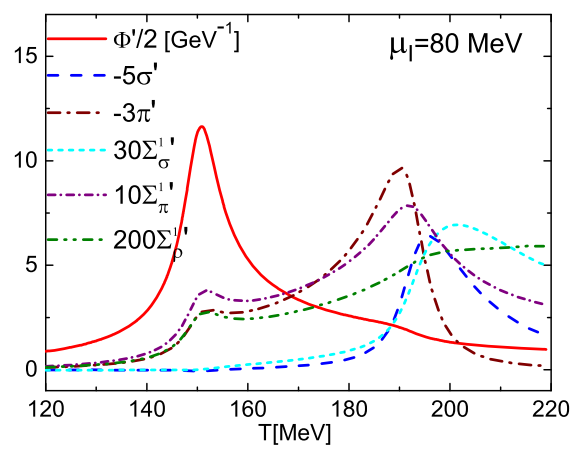

(d)

FIG. 11. Similar to Fig. 10, but for $T$-derivatives of the Polyakov loop, the sigma and pion condensates, the dual sigma and pion condensates, and the dual isospin density as functions of $T$.

$-\pi^{\prime}, \Sigma_{\pi}^{1 \prime}$, and $\Phi$ / each have one peak (and a bump near $T_{c}^{\chi}$ ), and the corresponding critical temperatures $T_{c}^{I_{3}}, T_{c}^{d \pi}$, and $T_{c}^{P}$ are almost identical. Note that $T_{c}^{d \pi} \approx T_{c}^{P}$ here is just a coincidence. We see that $-\sigma^{\prime}$ and $\Sigma_{\sigma}^{1 \prime}$ each have a dip at $T_{c}^{I_{3}}$. Compared to Figs. 11(a) and 11(b) indicates that the chiral restoration, the deconfining transition, and the melting of the pion condensate all become milder and the corresponding critical temperatures become smaller due to the improved unquenching effect. We see that the separation between $T_{c}^{P}$ and $T_{c}^{I_{3}}$ gets larger, but $T_{c}^{d \pi} \approx T_{c}^{I_{3}}$ still holds in Fig. 11(b). The eMF results shown in Figs. 11(c) and 11(d) display that $T_{c}^{d \pi}$ is considerably larger than $T_{c}^{P}$ but still equal to $T_{c}^{I_{3}}$. In contrast to Fig. 11(c), the peak of $\Sigma_{\pi}^{1 \prime}\left(\pi^{\prime}\right)$ near $T_{c}^{P}$ is significantly lower than that near $T_{c}^{I_{3}}$ (at $T_{c}^{I_{3}}$ ) due to the improved quark backreaction effect. Thus, all panels in Fig. 11 indicate that $T_{c}^{d \pi}$ just denotes the melting temperature of pion condensate, even though $\Sigma_{\pi}^{1}$ behaves like the Polyakov loop. We see that the $T$-dependences of the dual pion condensate and its $T$-derivative shown in Figs. 10 and 11 are similar to those of the dual sigma condensate and its slope displayed in Figs. 3 and 4, respectively. This is also in agreement with the PNJL results in Ref. [27].

Let us turn to the quantity $\mathrm{D} \rho \mathrm{C}$. We use $T_{c}^{\rho}$ to denote the highest peak of $\Sigma_{\rho}^{1 \prime}$. Figure 11(a) displays that $\Sigma_{\rho}^{1 \prime}$ peaks near $T_{c}^{P}$ (or $T_{c}^{I_{3}}$ ) and $T_{c}^{\chi}$, and the first peak is only slightly higher than the second, and thus $T_{c}^{\rho} \approx T_{c}^{P}$ or $T_{c}^{\rho} \approx T_{c}^{I_{3}}$. However, Fig. 11(b) shows clearly that $T_{c}^{\rho} \approx T_{c}^{\chi}$, since the deconfining transition becomes smoother. So, the sMF calculations suggest that the $\mathrm{D} \rho \mathrm{C}$ is more sensitive to chiral or deconfining transition depending on model parameters. The eMF results in Figs. 11(c) and 11(d) show that the $\mathrm{D} \rho \mathrm{C}$ is more sensitive to chiral transition: we see that the slope of $\Sigma_{\rho}^{1}$ at $T_{c}^{\chi}$ is obviously larger than that at $T_{c}^{P}$, but there is no obvious peak near $T_{c}^{\chi}$, since the slope still increases slowly with $T$ for $T>T_{c}^{\chi}$.

\section{Dependence on the parametrization}

To check the impact of the parametrization, we also study the $\mathrm{D} \sigma \mathrm{C}$ in $\mathrm{PL} \sigma \mathrm{M}$ using the improved polynomial glue potential $\mathcal{U}_{\text {glue }}^{\mathrm{pol}}$, which was adopted in Ref. [31]. Following Ref. [31], the dimensional regularization is employed, and the parameters $m_{q}^{0}=300 \mathrm{MeV}, m_{\sigma}=$ $540 \mathrm{MeV}$, and $T_{c}^{\text {glue }}=210 \mathrm{MeV}$ are chosen in our eMF calculation.

In Fig. 12, we show the resulting Polyakov loop, sigma condensate, and $\mathrm{D} \sigma \mathrm{C}$ and their $T$-derivatives as functions of $T$ for zero $\mu_{I}$. Note that besides the case with $m_{\sigma}=540 \mathrm{MeV}$, the eMF results for $m_{\sigma}=500 \mathrm{MeV}$ are 


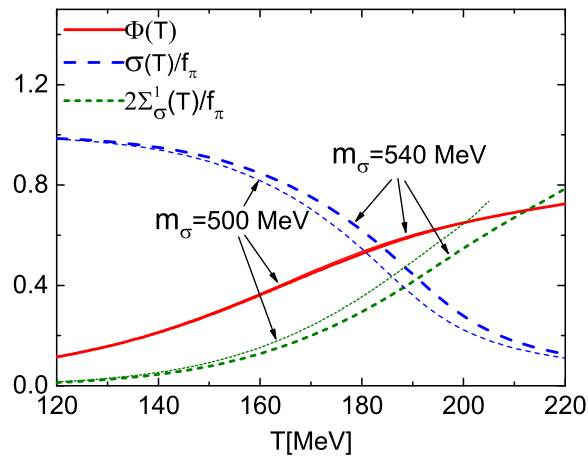

(a)

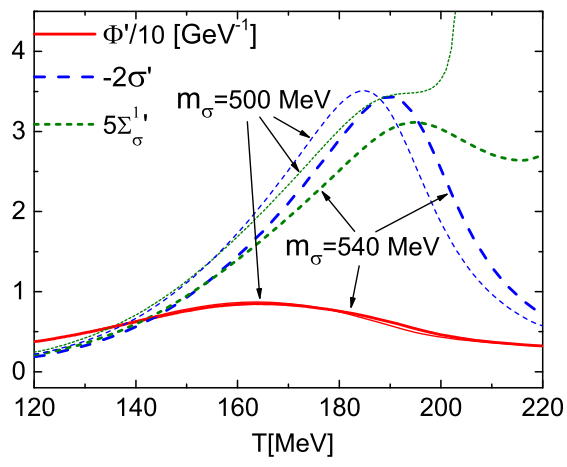

(b)

FIG. 12. (a) T-dependences of the eMF results for the Polyakov loop, the sigma condensate, and the dual sigma condensate, and (b) their $T$-derivatives at zero $\mu_{I}$ in the two-flavor PL $\sigma \mathrm{M}$ using the improved polynomial glue potential, $\mathcal{U}_{\mathrm{glue}}^{\text {pol }}$. The dimensional regularization is adopted, and $T_{c}^{\text {glue }}=210 \mathrm{MeV}$ and $m_{\sigma}=540 \mathrm{MeV}$ are chosen according to Ref. [31] (thick lines). The eMF results for $m_{\sigma}=500 \mathrm{MeV}$ are also shown for comparison (thin lines); the dual sigma condensate and its slope are plotted only up to $T=204 \mathrm{MeV}$ in this case, since $\sigma(\phi)$ becomes complex near the boson-like region $(\phi \sim 2 \pi, 0)$ for $T \gtrsim 204 \mathrm{MeV}$.

also displayed in Fig. 12 for comparison, and the sigma mass dependence will be discussed in the next section. Differently from results using $\mathcal{U}_{\text {glue }}^{\text {log }}$, Fig. 12 (a) shows that $\Phi$ increases more smoothly with $T$ near $T_{c}^{P}$. Consequently, $\Phi^{\prime},-\sigma^{\prime}$, and $\Sigma_{\sigma}^{1 \prime}$ each only peak near $T_{c}^{\chi}$, as displayed in Fig. 12(b). We thus still obtain $T_{c}^{d c} \approx T_{c}^{\chi}$, since $\Sigma_{\sigma}^{1 \prime}$ has no peak near $T_{c}^{P}$. The eMF results using the polynomial glue potential strongly suggest that the $\mathrm{D} \sigma \mathrm{C}$ cannot be used to indicate the deconfining transition. This conclusion is also robust in the sMF calculation using $\mathcal{U}_{\text {glue }}^{\text {pol }}$ due to the similar deconfining transition but the steeper chiral transition.

Similarly, we verify that the $T$-derivatives of $\mathrm{D} \sigma \mathrm{C}, \mathrm{D} \pi \mathrm{C}$, and $\mathrm{D} \rho \mathrm{C}$ have no peaks induced by the Polyakov loop when $\mathcal{U}_{\text {glue }}^{\text {pol }}$ is used for $\mu_{I}=80 \mathrm{MeV}$. Thus, in the polynomial parametrization of the glue potential, none of the three dual condensates obtained in PL $\sigma \mathrm{M}$ can be used as indicators of deconfining transition.

\section{Dependence on the sigma meson mass}

The sigma meson, $f_{0}(500)$ [previously denoted $\left.f_{0}(600)\right]$ is a rather broad resonance, $(400-550)-$ $i(200-350) \mathrm{MeV}$. In Ref. [30], $m_{\sigma}=(400-600) \mathrm{MeV}$ is considered as the reasonable range in the sMF calculation. We stress that our main conclusion will not change if a smaller $m_{\sigma}$ is adopted in sMF calculations. It is well known that decreasing $m_{\sigma}$ in $(\mathrm{P}) \mathrm{L} \sigma \mathrm{M}$ makes the chiral transition steeper, and thus the $\mathrm{D} \sigma \mathrm{C}$ should increases more swiftly near $T_{c}^{\chi}$. As demonstrated in Ref. [30], the deconfining transition region shrinks with decreasing $m_{\sigma}$, but decreasing $T_{c}^{\text {glue }}$ broadens it towards lower temperatures. Thus, the influence of decreasing $m_{\sigma}$ on the evolution of the Polyakov loop is mild. This means that the highest peak of the $\mathrm{D} \sigma \mathrm{C}$ slope in the sMF calculation should still be near $T_{c}^{\chi}$.
Actually, it was found in Ref. [30] that in order to reproduce the lattice pressure, the $T_{c}^{\text {glue }}$ should be lowered when decreasing $m_{\sigma}$ and vice versa: besides the parameter set $m_{\sigma}=500 \mathrm{MeV}$ and $T_{c}^{\text {glue }}=210 \mathrm{MeV}$, the optimal $T_{c}^{\text {glue }}$ for $m_{\sigma}=400(600) \mathrm{MeV}$ is $180(250) \mathrm{MeV}$ in the $2+1$ flavor case with the improved glue potential $\mathcal{U}_{\text {glue }}^{\text {log }}$. In Fig. 13, we show the sMF results for $T$-derivatives of the Polyakov loop, the sigma condensate, and the dual sigma condensates as functions of $T$ at vanishing $\mu_{I}$ in the twoflavor PL $\sigma \mathrm{M}$ using the above three sets of parameters. As expected, we see that with decreasing $m_{\sigma}, T_{c}^{\chi}$ moves towards lower temperatures and the chiral transition become steeper. In all three cases, the peak of $\Sigma_{\sigma}^{1 \prime}$ near $T_{c}^{\chi}$ is significantly higher than that near $T_{c}^{P}$, and thus the relation $T_{c}^{d \sigma} \approx T_{c}^{\chi}$ always holds. Actually, comparing Fig. 4(b) with Fig. 13(b) (both panels share the same $T_{c}^{\text {glue }}$ ), we can see that the chiral transition is quite sensitive to the choice of $m_{\sigma}$, but the conclusion $T_{c}^{d \sigma} \approx T_{c}^{\chi}$ is not. Similarly, comparing Fig. 4(b) with Fig. 13(c) (both panels share the same $m_{\sigma}$ ), we can see that the deconfining transition is quite sensitive to the choice of $T_{c}^{\text {glue }}$, but the conclusion $T_{c}^{d \sigma} \approx T_{c}^{\chi}$ is not.

In the eMF calculation, we have studied cases with $m_{\sigma}=$ 540 and $600 \mathrm{MeV}$, which is near the upper mass limit of $f_{0}(500)$. We have also tried to perform the eMF calculation at zero $\mu_{I}$ for smaller sigma meson masses. In Fig. 12(b), one can see that the peak of $\Sigma_{\sigma}^{1 \prime}$ near $T_{c}^{\chi}$ tends to vanish when the sigma meson mass is reduced from $540 \mathrm{MeV}$ to $500 \mathrm{MeV}$. Actually, the values of $\sigma(\phi)$ around $\phi \sim 2 \pi$ and 0 become complex when $T \gtrsim 204 \mathrm{MeV}$ for $m_{\sigma}=500 \mathrm{MeV}$. We find that near the boson-like region $(\phi \sim 2 \pi, 0), \sigma(\phi)$ has no real solutions about $m_{\sigma}<500 \mathrm{MeV}$ when $T$ is near and above $T_{c}^{\chi}$, no matter what logarithmic or polynomial glue potential is used. Thus, in this case, the $\mathrm{D} \sigma \mathrm{C}$ cannot be calculated 


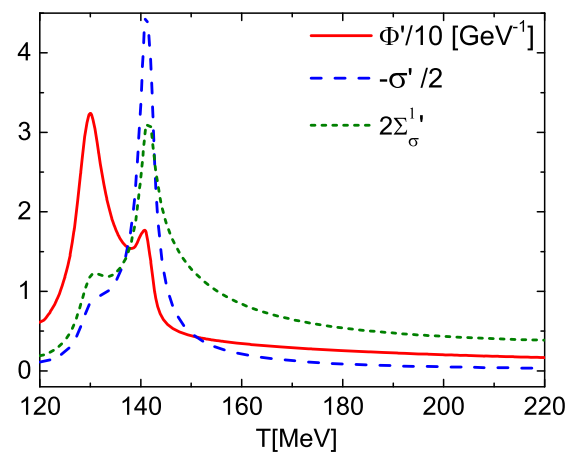

(a)

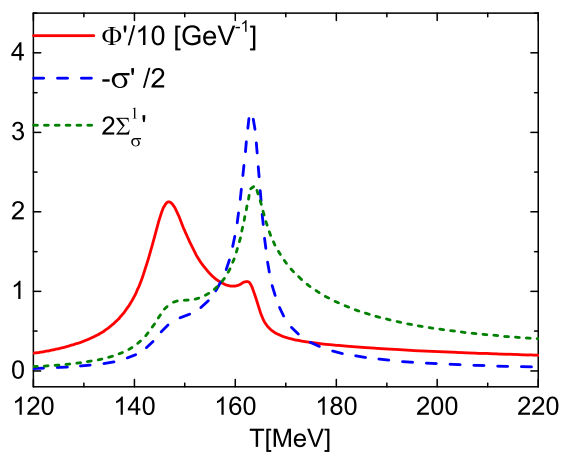

(b)

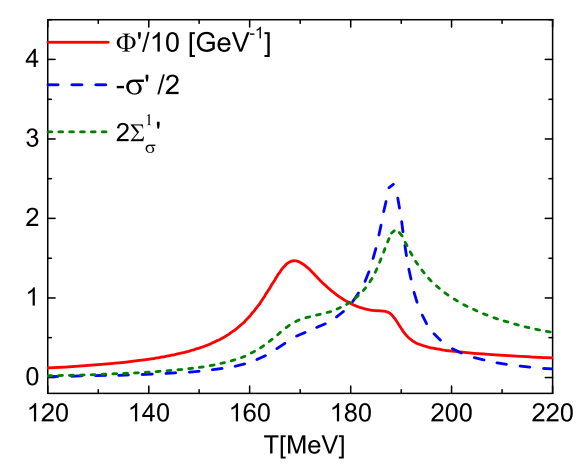

(c)

FIG. 13. Similar to Fig. 4(b), but for (a) $m_{\sigma}=400 \mathrm{MeV}$ and $T_{c}^{\text {glue }}=180 \mathrm{MeV}$, (b) $m_{\sigma}=500 \mathrm{MeV}$ and $T_{c}^{\text {glue }}=210 \mathrm{MeV}$, and (c) $m_{\sigma}=600 \mathrm{MeV}$ and $T_{c}^{\text {glue }}=250 \mathrm{MeV}$.

according to the definition in Eq. (24). This may reflect some limitation of the current method for the calculation of the dual observables, and we do not address this issue in this paper. Note that there is no such problem in the sMF calculation for $m_{\sigma}<500 \mathrm{MeV}$, as shown in Fig. 13.

On the other hand, we also perform the sMF (eMF) calculation using $m_{\sigma}=500(700) \mathrm{MeV}$ at $\mu_{I}=80 \mathrm{MeV}$. We confirm that the obtained results are qualitatively consistent with the corresponding sMF (eMF) calculations for $m_{\sigma}=600 \mathrm{MeV}$ shown in Figs. 10 and 11.

\section{E. Discussions}

Our calculations suggest that the slope of the dual sigma condensate at zero $\mu_{I}$ in PL $\sigma \mathrm{M}$ depends on the parametrization of the glue potential: It exhibits double peaks for the logarithmic parametrization, and the highest one is determined by chiral transition rather than the increase of the Polyakov-loop expectation value; on the other hand, it has only one peak which is determined by chiral transition for the polynomial parametrization. As a function of $T$, the slope of the dual sigma condensate behaves like the $T$-derivative of the minus sigma condensate rather than that of the Polyakov loop. Namely, the dual sigma condensate just reflects the same phase transition information as the sigma condensate does. In this sense, it seems that the dual meson condensates obtained in the two-flavor PL $\sigma \mathrm{M}$ of QCD are not qualified order parameters for deconfinement, even when the center symmetry is considered. This conclusion is consistent with (P)NJL studies [25-27]. Our study suggests that this conclusion is favored by the quark-improved glue potential. Moreover, such a conclusion is also verified at finite $\mu_{I}$.

The similar results in PL $\sigma \mathrm{M} / \mathrm{PNJL}$ may be indicative for QCD. First, the center symmetry breaking is large due to the light quarks. So it is very likely that some dual observables, such as the DPL or $\mathrm{D} \sigma \mathrm{C}$, are insensitive to deconfinement or Polyakov loop, unless the dynamical quarks are heavy enough. Second, formally, the definition of the DPL $(\mathrm{D} \sigma \mathrm{C})$ is naturally related to the quark (sigma) condensate. Thus, it is not strange that the DPL (D $\sigma \mathrm{C})$ is more sensitive to the chiral transition. Such a viewpoint is supported by the recent study of Dirac-mode expansion at finite imaginary chemical potential [54]: it shows that even VEVs of some quark bilinears can be expressed as the Polyakov loop and its conjugate for large quark masses; the quark number density (also the quark condensate) is still strongly dependent on low-lying Dirac-modes for small quark masses. ${ }^{9}$ Thus, it might be misleading to conclude

\footnotetext{
${ }^{9}$ It is reported in Ref. [54] that the sign of the quark number density is insensitive to low-lying Dirac modes, which supports the quark number holonomy [10] as the deconfinement indicator.
} 
the coincidence of chiral restoration and deconfinement through studying the DPL $(\mathrm{D} \sigma \mathrm{C})$.

Of course, the PL $\sigma \mathrm{M}$ and PNJL are just simple models, which may only partially reflect the possible relation between the (dynamically) center symmetry breaking and a dual observable existing in QCD. Especially, as modified chiral models PL $\sigma \mathrm{M}$ and PNJL are still more sensitive to chiral dynamics by construction, even the Polyakov-loop dynamics is included by considering the improved quark backreaction effect. So, the conclusion obtained in $(\mathrm{P}) \mathrm{L} \sigma \mathrm{M} /(\mathrm{P}) \mathrm{NJL}$ that the DPL or $\mathrm{D} \sigma \mathrm{C}$ may only be suitable to indicate chiral transition may not be really true in QCD. In addition, the investigations in PL $\sigma \mathrm{M}$ and PNJL do not exclude the possibility that some dual observables may be sensitive to deconfinement but insensitive to chiral transition.

In addition, there exists uncertainty on how to calculate the dual quark condensate. As mentioned, $\sigma(\phi)$ in full QCD has the Roberge-Weiss symmetry, which leads to zero $\Sigma_{\sigma}^{(1)}$ according to the integration in Eq. (24). One way to evade this problem is to break the Roberge-Weiss symmetry by hand, and the ordinary method is to first fix the gauge configuration under the antiperiodic boundary condition and then use it for other $\phi$ [15]. However, there is no justification for such a method so far. To avoid this uncertainty, it was proposed recently to use the RobergeWeiss periodic $\sigma(\phi)$ to perform the integration (24) with a modified integral range $\phi \in(-\pi / 3, \pi / 3)$ in Ref. [49]. The PNJL study suggests that the resulting dual quark condensate $\Sigma^{(1)}(T)$ behaves like the quark condensate, but $\Sigma^{(3)}(T)$ behaves like the Polyakov loop (for more details, see Ref. [49]).

Generally, deconfinement is associated with the liberation of degrees of freedom, manifested by the rapid rise in bulk thermodynamical quantities, such as the pressure, energy density, etc. Among them, the appropriate combinations of fluctuations and correlations of different conserved quantum numbers-for example, $\chi_{2}^{B}-\chi_{4}^{B}$ and $\chi_{31}^{B S}-\chi_{11}^{B S}$-directly probe the liberation of quark degrees of freedom $[55,56]$. It is interesting to study whether dual observables constructed from these bulk thermodynamical quantities are sensitive to deconfinement. There is a discussion on the dual pressure as the order parameter in Ref. [57]. Further investigation on this topic is needed which is beyond the scope of this paper.

Note that recent lattice calculations $[3,4]$ show that in the temperature region where the quark condensate drops rapidly, the renormalized Polyakov loop is still very small $\left(\sim 0.1\right.$ near $\left.T_{c}^{\chi}\right)$ and changes quite mildly. This implies that there might be no obvious connection between the chiral and deconfining transitions described in terms of these quantities. In contrast, the Polyakov loop calculated in effective models is relatively large near $T_{c}^{\chi}$, reaching unity quickly. This big discrepancy has been discussed recently by Pisarski and Skokov in the chiral matrix model [58], ${ }^{10}$ and the reason is still unclear. It was argued that the difference can be partially attributed to the fact that $\Phi\left(\left\langle A_{0}\right\rangle\right)>\langle\Phi\rangle$ [30]. On the other hand, the entropy of static quarks calculated in lattice simulation [4] suggests that the deconfinement and chiral transitions happen at similar temperatures. Thus, whether the chiral and deconfining transitions have a close relation or not is still a subtle problem, and the sensitive probe of deconfinement needs to be further investigated.

\section{CONCLUSION}

Dual meson condensates as the possible order parameters for center symmetry are tested in PL $\sigma \mathrm{M}$. To our knowledge, this is the first paper employing the PLoM at finite imaginary chemical potential. We mainly study the thermal property of the dual sigma condensate. The dual pion and vector meson condensates at $\mu_{I}>m_{\pi} / 2$ are also investigated. We focus on roles of the fermion vacuum loop contribution, the parametrization of the glue potential, and the improved unquenching effect.

At zero density, we find that the $\mathrm{D} \sigma \mathrm{C}$ really behaves like the thin or dressed Polyakov loop. The $T$-derivative of the $\mathrm{D} \sigma \mathrm{C}$ may have double or single peaks, which depends on the parametrization of the glue potential. We verify that the peak structure of the slope of the $\mathrm{D} \sigma \mathrm{C}$ is quite similar to that of the sigma condensate but not the Polyakov loop. So, the crossover temperature determined by the $\mathrm{D} \sigma \mathrm{C}$ just indicates chiral restoration rather than deconfining transition. This conclusion is favored by the quark-improved glue potential, since the deconfining crossover is softened, no matter whether the fermion vacuum contribution is included or not. Qualitatively, the evolution of the $\mathrm{D} \sigma \mathrm{C}$ with $T$ in PL $\sigma \mathrm{M}$ is consistent with that obtained in the $\mathrm{L} \sigma \mathrm{M}$ without the glue potential.

For $\mu_{I}>m_{\pi} / 2$, the $\mathrm{D} \sigma \mathrm{C}$ shows abnormal thermal behavior: it first decreases or almost remains unchanged with $T$ and then increases, which is distinct with the Polyakov loop. We reveal that with increasing $T$, the $\mathrm{D} \sigma \mathrm{C}$ increases if $\sigma$ decreases, and vice versa. The anomaly gives further evidence that the $\mathrm{D} \sigma \mathrm{C}$ is quite sensitive to chiral dynamics but not center symmetry. In contrast, the $\mathrm{D} \pi \mathrm{C}$ and $\mathrm{D} \rho \mathrm{C}$ still exhibit similar $T$-dependence to the Polyakov loop. We confirm that the maximum slope of the $\mathrm{D} \pi \mathrm{C}$ does not indicate the deconfining crossover, but the restoration of $U(1)_{I_{3}}$ symmetry. Without the fermion vacuum contribution, whether the $\mathrm{D} \rho \mathrm{C}$ is more sensitive to chiral or deconfining transition depends on the model parameters: it is more sensitive to chiral transition when the improved unquenching effect is considered; when

\footnotetext{
${ }^{10}$ The deconfining crossover temperature $T_{c}^{P}$ extracted from the Polyakov loop is almost the same as $T_{c}^{\chi}$ in this model, which can be achieved by using a quite small $m_{\sigma}(<400 \mathrm{MeV})$.
} 
including the Dirac sea contribution, the rapid rise of the $\mathrm{D} \rho \mathrm{C}$ is also more sensitive to chiral restoration.

We thus conclude that the dual meson condensates are not appropriate order parameters for deconfinement in $\mathrm{PL} \sigma \mathrm{M}$ (also in $\mathrm{L} \sigma \mathrm{M}$ ). Our results are qualitatively consistent with the calculations in NJL at zero density [25,26] and PNJL at $\mu_{I}>m_{\pi} / 2$ [27]. We argue that the reason can be attributed to either the fact that the explicit center symmetry breaking is large due to light quarks and thus not all the dual observables are qualified order parameters for deconfinement, or the limitation of simple models in which some intrinsic connection between the center symmetry and a dual observable is ignored.

\section{ACKNOWLEDGMENTS}

Z.Z. was supported by the National Natural Science Foundation of China (Grants No. 11875127 and No. 11275069).
[1] Y. Aoki, S. Borsanyi, S. Durr, Z. Fodor, S. D. Katz, S. Krieg, and K. K. Szabo, J. High Energy Phys. 06 (2009) 088.

[2] S. Borsanyi, Z. Fodor, C. Hoelbling, S. D. Katz, S. Krieg, C. Ratti, and K. K. Szabó (Wuppertal-Budapest Collaboration), J. High Energy Phys. 09 (2010) 073.

[3] A. Bazavov and P. Petreczky, Phys. Rev. D 87, 094505 (2013).

[4] A. Bazavov, N. Brambilla, H.-T. Ding, P. Petreczky, H.-P. Schadler, A. Vairo, and J. H. Weber, Phys. Rev. D 93, 114502 (2016).

[5] K. Fukushima and V. Skokov, Prog. Part. Nucl. Phys. 96, 154 (2017).

[6] H. Suganuma, S. Sasaki, and H. Toki, Nucl. Phys. B435, 207 (1995).

[7] M. N. Chernodub, Y. Nakagawa, A. Nakamura, T. Saito, and V. I. Zakharov, Phys. Rev. D 83, 114501 (2011).

[8] P. M. Lo, B. Friman, O. Kaczmarek, K. Redlich, and C. Sasaki, Phys. Rev. D 88, 074502 (2013).

[9] A. Miyahara, Y. Torigoe, H. Kouno, and M. Yahiro, Phys. Rev. D 94, 016003 (2016).

[10] K. Kashiwa and A. Ohnishi, Phys. Rev. D 93, 116002 (2016).

[11] K. Kashiwa and A. Ohnishi, Phys. Lett. B 772, 669 (2017).

[12] M. Mitter, M. Hopfer, B.-J. Schaefer, and R. Alkofer, Phys. Lett. B 777, 114 (2018).

[13] C. Gattringer, Phys. Rev. Lett. 97, 032003 (2006).

[14] E. Bilgici, F. Bruckmann, C. Gattringer, and C. Hagen, Phys. Rev. D 77, 094007 (2008).

[15] E. Bilgici, F. Bruckmann, J. Danzer, C. Gattringer, C. Hagen, E. M. Ilgenfritz, and A. Maas, Few-Body Syst. 47, 125 (2010).

[16] B. Zhang, F. Bruckmann, C. Gattringer, Z. Fodor, and K. K. Szabo, AIP Conf. Proc. 1343, 170 (2011).

[17] J. Braun, L. M. Haas, F. Marhauser, and J. M. Pawlowski, Phys. Rev. Lett. 106, 022002 (2011).

[18] C. S. Fischer, Phys. Rev. Lett. 103, 052003 (2009).

[19] C. S. Fischer and J. A. Mueller, Phys. Rev. D 80, 074029 (2009).

[20] C. S. Fischer, J. Luecker, and J. A. Mueller, Phys. Lett. B 702, 438 (2011).

[21] C. S. Fischer, A. Maas, and J. A. Muller, Eur. Phys. J. C 68 , 165 (2010).
[22] K. Kashiwa, H. Kouno, and M. Yahiro, Phys. Rev. D 80, 117901 (2009).

[23] R. Gatto and M. Ruggieri, Phys. Rev. D 82, 054027 (2010).

[24] T. K. Mukherjee, H. Chen, and M. Huang, Phys. Rev. D 82, 034015 (2010).

[25] S. Benic, Phys. Rev. D 88, 077501 (2013).

[26] F. Marquez, A. Ahmad, M. Buballa, and A. Raya, Phys. Lett. B 747, 529 (2015).

[27] Z. Zhang and Q. Miao, Phys. Lett. B 753, 670 (2016).

[28] K. Fukushima, Phys. Lett. B 591, 277 (2004).

[29] S. Roessner, C. Ratti, and W. Weise, Phys. Rev. D 75, 034007 (2007).

[30] L. M. Haas, R. Stiele, J. Braun, J. M. Pawlowski, and J. Schaffner-Bielich, Phys. Rev. D 87, 076004 (2013).

[31] T. K. Herbst, M. Mitter, J. M. Pawlowski, B. J. Schaefer, and R. Stiele, Phys. Lett. B 731, 248 (2014).

[32] R. Stiele, E. S. Fraga, and J. Schaffner-Bielich, Phys. Lett. B 729, 72 (2014).

[33] R. Stiele and J. Schaffner-Bielich, Phys. Rev. D 93, 094014 (2016).

[34] P. Kovács, Z. Szép, and G. Wolf, Phys. Rev. D 93, 114014 (2016).

[35] J. M. Torres-Rincon and J. Aichelin, Phys. Rev. C 96, 045205 (2017).

[36] D. Fuseau, T. Steinert, and J. Aichelin, Phys. Rev. C 101, 065203 (2020).

[37] B. J. Schaefer, J. M. Pawlowski, and J. Wambach, Phys. Rev. D 76, 074023 (2007).

[38] M. Buballa and S. Carignano, Prog. Part. Nucl. Phys. 81, 39 (2015).

[39] J. O. Andersen, W. R. Naylor, and A. Tranberg, Rev. Mod. Phys. 88, 025001 (2016).

[40] O. Scavenius, A. Mocsy, I. N. Mishustin, and D. H. Rischke, Phys. Rev. C 64, 045202 (2001).

[41] V. Skokov, B. Friman, E. Nakano, K. Redlich, and B.-J. Schaefer, Phys. Rev. D 82, 034029 (2010).

[42] H. Ueda, T.Z. Nakano, A. Ohnishi, M. Ruggieri, and K. Sumiyoshi, Phys. Rev. D 88, 074006 (2013).

[43] J.I Kapusta and C. Gale, Finite-Temperature Field Theory: Principles and Applications, 2nd ed. (Cambridge University Press, Cambridge, England, 2006), p. 4228.

[44] K. Kamikado, N. Strodthoff, L. von Smekal, and J. Wambach, Phys. Lett. B 718, 1044 (2013). 
[45] Z. Zhang and Y.-X. Liu, Phys. Rev. C 75, 064910 (2007).

[46] D. T. Son and M. A. Stephanov, Phys. Rev. Lett. 86, 592 (2001).

[47] P. Cea, L. Cosmai, M. D’Elia, A. Papa, and F. Sanfilippo, Phys. Rev. D 85, 094512 (2012).

[48] M. D'Elia and F. Sanfilippo, Phys. Rev. D 80, 111501 (2009).

[49] K. Kashiwa and H. Kouno, Phys. Rev. D 100, 094023 (2019).

[50] Z. Zhang and H. P. Su, Phys. Rev. D 89, 054020 (2014).

[51] N. Strodthoff, B. J. Schaefer, and L. von Smekal, Phys. Rev. D 85, 074007 (2012).
[52] L. y. He, M. Jin, and P. f. Zhuang, Phys. Rev. D 71, 116001 (2005).

[53] Z. Zhang and Y.x. Liu, Phys. Rev. C 75, 035201 (2007).

[54] T. M. Doi and K. Kashiwa, arXiv:1706.00614.

[55] A. Bazavov et al., Phys. Rev. Lett. 111, 082301 (2013).

[56] R. Bellwied, S. Borsanyi, Z. Fodor, S. D. Katz, and C. Ratti, Phys. Rev. Lett. 111, 202302 (2013).

[57] K. Kashiwa, arXiv:1603.04958.

[58] R. D. Pisarski and V. V. Skokov, Phys. Rev. D 94, 034015 (2016). 